\title{
POBREZA, DESIGUALDAD Y SEGREGACIÓN EN LA REGIÓN METROPOLITANA*
}

\author{
Claudio A. Agostini
}

En general, hay consenso respecto a cómo medir pobreza y desigualdad. Es por ello que existe un grado importante de acuerdo sobre su evolución en el tiempo, tanto en Chile como en la Región Metropolitana. Sin embargo, no existe igual grado de acuerdo respecto a la segregación. No hay una definición clara de qué es segregación ni cómo medirla, ni hay datos validados que permitan ver su evolución en el tiempo. No obstante, la ciudad de Santiago suele ser percibida como una urbe muy segregada y se piensa que ello tiene efectos negativos. En general, no hay buenos argumentos para concluir que la segregación geográfica es mala per se y sí hay casos en que puede ser neutra o incluso buena. La evidencia en la literatura económica muestra consistentemente dos casos en los cuales sí hay efectos negativos: minorías raciales y pobres. En el caso de Chile, existe también algo de evidencia de que la segregación residencial tiene efectos negativos en

Claudio Agostini. Doctor en Economía, University of Michigan. Profesor asociado, ILADES-Universidad Alberto Hurtado.

*Agradezco los comentarios de Alexander Galetovic, Pablo Allard y Manuel Tironi. En especial, agradezco a Magdalena Opazo por su excelente, riguroso y dedicado apoyo en la investigación.

Estudios Públicos, 117 (verano 2010). 
los más pobres. Por esta razón, un análisis relevante para la ciudad de Santiago debiera intentar responder qué tan segregada es la ciudad en términos de ingreso y, especialmente, de pobreza. El objetivo principal de este trabajo es contribuir a responder dicha pregunta. La evidencia empírica que se presenta muestra que la realidad es bastante menos dramática que la percepción que se tiene de Santiago: la ciudad se ha vuelto menos segregada y algunas afirmaciones sobre ella no se ven confirmadas en los datos. La calidad de vida ha mejorado mucho y las diferencias entre comunas en la Región Metropolitana se han reducido en forma significativa. Una excepción importante es el nivel de capital humano, donde hay diferencias importantes. Es por ello que tanto la pobreza como la educación debieran tener mayor prioridad en el debate público que la segregación.

Palabras clave: pobreza; desigualdad; segregación; Santiago.

Clasificación JEL: I32, D31, D63, O10, O15.

"En el nuevo contexto de globalización de las economías, la segregación residencial se está volviendo 'maligna' en nuestras ciudades."

(Francisco Sabatini, Gonzalo Cáceres y Jorge Cerda en revista EURE, 2001)

\section{Introducción}

$\boldsymbol{H}_{\mathrm{n}}$ n general, existe bastante consenso respecto a qué son la pobreza y la desigualdad y cómo medirlas. En el caso de la pobreza, la definición conceptual se refiere a personas u hogares cuyo ingreso es menor que el necesario para poder consumir una canasta de bienes que satisfaga las necesidades mínimas (consumo básico). Si bien se puede medir la pobreza también en términos de gasto, el estándar es utilizar ingreso, ya que es una medida razonable de las oportunidades que tiene una familia y no está influida por las decisiones específicas de consumo que tome (Atkinson, 2008). Es así como se identifica como pobres a aquellos hogares que se encuentran bajo el nivel de ingreso que determina la condición de pobreza (bajo la línea de pobreza). 
En el caso de la desigualdad, el objetivo es poder comparar distribuciones del ingreso, per cápita o del hogar, en forma cuantitativa. La medida más utilizada para cuantificar desigualdad es el coeficiente de Gini, pero también se utiliza la proporción del ingreso total nacional que poseen distintos subgrupos de la población, en particular los distintos deciles o quintiles (Glaeser, 2005).

También hay un grado importante de acuerdo respecto a su evolución en el tiempo en Chile en general y en la ciudad de Santiago en particular. Si bien puede haber algún grado de discrepancia en los detalles metodológicos en el cálculo y actualización de las cifras oficiales, no hay discrepancias respecto a que la pobreza ha disminuido en forma importante en Chile durante los últimos 20 años, mientras que los niveles de desigualdad se han mantenido relativamente constantes en el mismo período.

No hay igual consenso en cuanto a la segregación. No existe una definición clara de qué es segregación, tampoco hay acuerdo en una forma de medirla y no hay datos validados que permitan ver su evolución en el tiempo.

La percepción general respecto a la ciudad de Santiago es que se trata de una ciudad muy segregada (Rodríguez, 2001; Vargas, 2006), lo cual tendría efectos negativos en sus habitantes y en el funcionamiento de la ciudad misma (Sabatini, 2006). En esta discusión se entiende por segregación, a partir de un concepto sociológico, la falta de interacción entre grupos sociales. De esta forma, la segregación geográfica implica una distribución no homogénea de distintos grupos sociales en un espacio físico, en este caso en la ciudad, y donde por grupo social se entiende nivel de ingreso o grupo socioeconómico.

Si consultamos el Diccionario de la Real Academia Española, segregar se define, en primer lugar, como "Separar o apartar algo de otra u otras cosas" y, en segundo lugar, como "Separar y marginar a una persona o a un grupo de personas por motivos sociales, políticos o culturales”. En ambas acepciones está implícita la idea de que la separación no fue voluntaria sino que forzada, y tal vez eso lleva a la connotación negativa que tiene la segregación.

Tal vez lo primero que es importante mencionar entonces, con el objeto de reducir la carga negativa que tiene la palabra "segregación", es que la segregación ocurre no sólo en un contexto de niveles de ingreso o grupos sociales. Hay segregación entre extranjeros y chilenos 
en la plaza de Armas, entre alumnos y profesores en una sala donde se presenta un seminario, entre personas de distintos países en una comida, en una conferencia, y entre distintas especies animales que ocupan un territorio geográfico. Estos ejemplos reflejan que la segregación geográfica no tiene por qué ser mala per se, sino que puede también ser neutra o incluso buena.

Si bien es difícil determinar empíricamente los efectos de la segregación residencial en distintas variables de éxito personal de sus habitantes, la literatura económica muestra consistentemente dos casos en los cuales hay efectos negativos: minorías raciales y pobres.

En el caso de minorías raciales, en particular de los negros, la evidencia muestra que la segregación espacial tiene efectos negativos en los resultados escolares, el empleo y la probabilidad de ser padre/ madre soltero(a) (Cutler y Glaeser, 1997). Adicionalmente, se generan externalidades negativas en oportunidades educacionales, barreras culturales, restricciones al flujo de capital, oportunidades de empleo y desincentivos para invertir en el stock de viviendas, las cuales restringen la movilidad social y reducen la calidad de vida (Vandell, 1995).

En el caso de las personas de menores ingresos, que es más relevante para la discusión sobre Santiago que el caso de minorías raciales, la evidencia es que los pobres que viven aislados en barrios pobres tienen una peor situación de pobreza que los pobres que viven en barrios más heterogéneos, lo que se traduce en peores resultados académicos, peor estado de salud y mayores tasas de criminalidad (Dawkins et al., 2005; Charles et al., 2004; Clapp y Ross, 2004; Burton, 2003; Logan y Messner, 1987). La explicación es que en barrios donde se concentra pobreza, en general, hay pocos modelos (role models) positivos, el crimen y las drogas son más frecuentes y visibles, los buenos trabajos pueden no inexistir o estar ubicados muy lejos y los servicios como la educación tienen menos financiamiento y son de inferior calidad (Yinger, 1979; Galster, 1991, 1992; Galster y Killen, 1995).

Para el caso de Chile, Larrañaga y Sanhueza (2007) muestran que la segregación residencial tiene efectos negativos en los más pobres y sus familias, haciendo que los hijos de esas familias tengan menores oportunidades de asistir a educación preescolar, mayor retraso escolar - tanto en términos de edad como en términos de grado- y mayor probabilidad de abandonar la escuela. Los resultados muestran 
también que aunque la segregación reduce las probabilidades de participación en la fuerza de trabajo, no afecta la probabilidad o el riesgo de maternidad adolescente, ni tampoco el estatus de salud de la población en edad de trabajar.

Dada esta evidencia, el análisis relevante para la ciudad de Santiago debiera intentar responder qué tan segregada es la ciudad en términos de ingreso y, especialmente, de pobreza. El objetivo principal de este trabajo es contribuir a responder dicha pregunta. Para ello, aquí se analizan primero cómo se mide la segregación y las ventajas y desventajas que tienen dichas medidas. Segundo, se discute lo que se sabe, se ha dicho y se ha medido de la segregación en Santiago, con el objeto de utilizar dicha discusión como base para examinar el análisis empírico posterior. Por último, se hace un análisis empírico de la segregación en la Región Metropolitana, su evolución en algunas dimensiones y se contrastan los resultados con las creencias que existen respecto a la ciudad.

\section{II. ¿Qué es y cómo medir la segregación?}

En la literatura económica, el concepto de segregación está relacionado con el funcionamiento del mercado de viviendas y la ubicación de los hogares en una zona urbana. Es así como la definición de "segregación residencial" corresponde a "un equilibrio en el mercado de viviendas caracterizado por disparidades en la ubicación física de hogares pertenecientes a diferentes grupos raciales, étnicos o socioeconómicos” (Vigdor, 2008). En este sentido, la segregación consiste simplemente en formas de desigual distribución de grupos de población en el territorio.

Dada esta definición, la segregación no es ni mala ni buena, sino que sólo refleja un equilibrio en el mercado de viviendas entre muchos equilibrios posibles. La única preocupación sería si dicho equilibrio es ineficiente desde el punto de vista de la asignación de los recursos o si es subóptimo desde el punto de vista del bienestar social. La ineficiencia en la asignación de recursos podría ocurrir si los hogares no tienen información completa para tomar sus decisiones de ubicación o si enfrentan restricciones de liquidez que les impiden endeudarse. Desde el punto de vista del bienestar social, el equilibrio puede ser subóptimo 
porque la segregación permite que los hogares de más altos ingresos contribuyan menos al financiamiento de bienes públicos locales que consumen en mayor proporción los hogares de menores ingresos, lo cual tendría efectos distributivos que pueden ser indeseables, dependiendo de las preferencias sociales por redistribución.

Más allá de las ineficiencias que podrían ocurrir en un equilibrio de segregación residencial, lo relevante es cómo poder medir cada equilibrio de tal forma de poder compararlos y cuantificar potenciales ineficiencias. En general, no es fácil construir un indicador de segregación y en la literatura se han hecho varios esfuerzos en esa dirección sin que haya consenso respecto a la mejor forma de medir la segregación.

El índice más conocido y utilizado es el Índice de Disimilitud o Índice de Duncan (Duncan y Duncan, 1955), que mide el porcentaje de un grupo que debe cambiarse de ubicación en cada subunidad geográfica para que en esa subunidad el porcentaje de ese grupo sea el mismo que el que se da en la unidad geográfica superior (por ejemplo, el porcentaje de miembros pobres que tendrían que irse a vivir a otro barrio para obtener una distribución homogénea de hogares pobres en una ciudad). El índice tiene valores entre 0 y 1, donde 0 es integración total y 1 es segregación total.

Lo interesante de este índice es que entrega una medida que permite comparar el nivel de segregación entre distintas ciudades y distintos países, si bien la definición del grupo que se está evaluando y la característica que se toma en cuenta para hacerlo son algo subjetivo y quedan a criterio del investigador.

Por otro lado, el Índice de Disimilitud no está exento de críticas. En particular, este índice tiene el problema de checkerboard, consistente en que las unidades geográficas (celdas en un tablero) pueden barajarse para obtener otra configuración espacial y el índice no cambia. Además el índice es sensible al tamaño de la unidad geográfica (celda) y aumenta cuando el tamaño de la celda disminuye, es decir, la medida de segregación a nivel comunal es menor que la medida a nivel de manzanas o barrios sólo por el hecho de que las manzanas y barrios tienen un tamaño menor que una comuna. Incluso más, la correlación entre el índice calculado a nivel de distrito censal y el índice calculado a nivel de manzana es sólo de 0,58 en 144 ciudades en Estados Unidos (Van Valey y Roof, 1976). Sin embargo, la crítica mayor al Índice de Disimilitud es que encuentra niveles de segregación distintos de cero 
incluso cuando las personas son asignadas aleatoriamente en distintos vecindarios de una ciudad (Fosset, 2005).

Un segundo índice también utilizado para medir segregación residencial es el Índice de Exposición/Aislamiento (Bell, 1954; Erbe, 1975), que mide la probabilidad promedio de contacto entre miembros de dos grupos distintos en una unidad geográfica (pobres y no pobres en una comuna por ejemplo) a partir de la proporción de personas de un grupo minoritario respecto a otro mayoritario que viven en la misma unidad geográfica ${ }^{1}$.

\section{Mediciones de segregación}

\section{Índice de Disimilitud:}

La expresión matemática para este índice es la siguiente:

$$
\mathrm{D}=\sum_{i=1}^{N}\left[\frac{t_{i}\left(p_{i}-p\right)}{2 T p(1-p)}\right]
$$

donde $t_{i}$ representa la población total en la subunidad geográfica i; $p_{i}$ es la cantidad de individuos del grupo que se desea investigar en la subunidad geográfica i, $T$ es la población total de la unidad geográfica superior y $p$ es la cantidad de personas del grupo en estudio en el total de la unidad geográfica superior.

\section{Índice de Exposición/Aislamiento:}

La expresión matemática de este índice es la siguiente:

$$
p^{*}=\sum_{i=1}^{N}\left[\frac{x_{i} y_{i}}{X t_{i}}\right]
$$

donde $x_{i}$ es el grupo minoritario e $y_{i}$ el grupo mayoritario en la subunidad i; $X$ es la población total del grupo minoritario; y $t_{i}$ es la población total en la subunidad $i$.

${ }^{1}$ El Índice de Aislamiento mide la probabilidad de contacto entre miembros de un mismo grupo en una unidad geográfica. Si se consideran sólo dos grupos el Índice de Exposición y el Índice de Aislamiento suman 1. 
Ambos índices consideran la segregación entendida como el grado de contacto entre distintos grupos sociales, pero son índices no espaciales ya que no consideran la localización espacial de los grupos en la unidad geográfica. En este sentido, aunque son ampliamente utilizados en la literatura académica, estos dos índices son complementarios de otras miradas que permiten analizar espacialmente la segregación.

\section{III. ¿Qué se dice y qué sabemos de la segregación residencial en Chile?}

Para algunos, la existencia de segregación en nuestras ciudades es simplemente una característica incuestionable de ellas y tendría algunos orígenes históricos. Sabatini, Cáceres y Cerda (2001), por ejemplo, mencionan que "el sello de las ciudades latinoamericanas es la segregación residencial a gran escala”, que se origina porque "a lo largo del siglo XX las familias de las elites se fueron concentrando por lo general en una sola zona de crecimiento que, en la forma de un cono, une el centro histórico con la periferia en una dirección geográfica definida”. En este contexto, para el caso de Chile un determinante fundamental de la segregación residencial sería la liberalización de los mercados de suelo, junto con los procesos de globalización económica y cultural. Estos procesos habrían llevado a una transformación del patrón tradicional de segregación residencial de las ciudades latinoamericanas, convirtiendo la distribución de las ciudades en lo que hoy conocemos. El paso ha sido desde un modelo de ciudades "compactas" al estilo europeo, donde los grupos de ingresos altos estaban en el centro de la ciudad junto con la mejor arquitectura y calidad de las construcciones, hacia un modelo en el que los grupos de mayores ingresos han tendido a urbanizar las periferias, se han creado zonas de acceso a bienes y servicios distintos y lejanos del centro urbano y ha habido un desplazamiento de las familias de ingresos medios de las zonas urbanas como consecuencia de los altos precios del suelo (Sabatini, 2006).

Es así como la visión que predomina, para Latinoamérica en general y para Chile en particular, es bastante negativa respecto a la evolución que ha tenido el desarrollo de las ciudades, sus niveles de segregación y los efectos negativos que ésta provocaría. Rodríguez (2001), a partir de datos del Censo de Población y Vivienda de 1992, 
concluye que Santiago es una ciudad segregada, con un nivel de segregación moderado pero estable. Sabatini, Cáceres y Cerda (2001) señalan que el patrón de segregación residencial tradicional de las ciudades chilenas, que es también el de la mayoría de las ciudades latinoamericanas, se está transformando desde hace un par de décadas en dos sentidos principales: está cambiando su “escala geográfica” y está aumentando su "malignidad". De igual forma, Sabatini (2006) señala que el problema es precisamente la "malignidad" de la segregación y los patrones que ésta ha ido desarrollando en la mayoría de las ciudades latinoamericanas, donde ha aumentado la escala e intensidad de la segregación objetiva junto a una dimensión subjetiva relacionada con estigmas territoriales. Vargas (2006) fundamenta su análisis sobre segregación residencial partiendo de la base que Santiago es de las ciudades más segregadas del mundo, lo que se confirmaría a partir de indicadores que difieren sustancialmente entre las comunas de mayor ingreso y las de menor ingreso.

Lamentablemente, esta visión negativa sobre la segregación residencial en Chile no siempre está bien respaldada con análisis estadísticos muy precisos, no siempre se consideran hipótesis alternativas que podrían explicar igual de exitosamente los datos observados, ni tampoco está siempre bien documentada. Rodríguez (2001) señala que "Santiago de Chile [es] considerada por muchos estudiosos como una de las ciudades más segregadas del planeta”. Sin embargo, no cita ni menciona ninguno de los "muchos estudiosos" ni tampoco ninguno de sus estudios. Sabatini (2006) menciona como un aspecto negativo de la segregación el que se configuren áreas de una ciudad que sean socialmente homogéneas y uno de los problemas en las ciudades en Latinoamérica es que las áreas habitadas por los más pobres son más socialmente homogéneas que las habitadas por las clases altas. Sin embargo, esto último puede simplemente deberse a que el número de pobres es muy superior al número de ricos y, por tanto, no es el resultado de un fenómeno de segregación.

La evidencia empírica en la literatura académica muestra una alta correlación positiva entre desigualdad y segregación ${ }^{2}$, y existe además una relación matemática entre ambas (Duncan y Duncan, 1955; Hutchens, 1991); por ello es que los indicadores de desigualdad entregan

\footnotetext{
${ }^{2}$ Véase, por ejemplo, Sethi Somanaathan (2004); Ross, Nobrega y Dunn (2004); Kremer y Maskin (1996).
} 
información también sobre segregación. Sin embargo, en la discusión en Chile no hay acuerdo en que eso sea cierto. Para Sabatini (2004), por ejemplo, la dimensión espacial es un componente esencial de la vida en sociedad, por lo que segregación y desigualdad no tienen una relación directa. La primera es un fenómeno espacial y la segunda un fenómeno social. La segregación residencial habría que entenderla entonces como la separación territorial o espacial entre personas o familias pertenecientes a un mismo grupo social y "presenta tres dimensiones: (a) la tendencia de ciertos grupos sociales a concentrarse en algunas áreas de la ciudad generando barrios donde, en el extremo, llegan a tener un claro predominio numérico, o en casos menos extremos, comparten en mayor grado con otros grupos; (b) la conformación de áreas con un alto grado de homogeneidad social; y (c) la percepción subjetiva que la gente tiene de la segregación 'objetiva' (las dos primeras dimensiones)”. La primera dimensión permite explicar la concentración espacial de las familias de la elite en ciertas zonas de las ciudades, mientras que la segunda dimensión permite explicar que aunque la concentración sea alta, en ese mismo espacio conviven familias de ingresos medios y bajos, por lo que la homogeneidad también es alta. Sin embargo, sería la tercera dimensión la que produce efectos sobre la desintegración social, ya que las familias pobres, al vivir en barrios pobres, sienten la marginalidad, “convirtiéndose así en un fenómeno real" (Sabatini, 2004).

De acuerdo a esta visión, que muchas veces se cometa el error de equiparar segregación a desigualdad tiene que ver con lo que vemos en la realidad: que el espacio donde se concentran las elites es de homogeneidad social, pero el espacio en el que se concentran los pobres no (Sabatini, Cáceres y Cerda, 2001). La segregación residencial que observamos sería un recurso complementario que utilizan los grupos sociales para mantener sus identidades, las que actualmente se encuentran en permanente riesgo debido a la movilidad social que existe al interior de las sociedades y por eso el componente subjetivo de la segregación sería especialmente importante de estudiar.

Es así que el hecho de que la segregación residencial se convierta en un problema social tiene que ver con el cambio en la escala geográfica experimentado y con la malignidad creciente de este fenómeno. A partir de esta idea Sabatini, Cáceres y Cerda (2001) estudian la segregación residencial socioeconómica en tres ciudades de Chile, 
con datos censales y una mirada sobre la ocupación del jefe de hogar, en Concepción y Valparaíso, y con datos de la Encuesta de Origen y Destino (1991) que contiene ingresos, en Santiago. De acuerdo a su análisis, la escala geográfica de la segregación estaría disminuyendo en aquellas zonas de la ciudad donde hay mayor dinamismo privado inmobiliario, pero estaría aumentando en aquellas zonas donde se están asentando las nuevas familias de bajos ingresos. El problema entonces sería que mientras más grandes sean las áreas homogéneas en pobreza, más se agravarían los problemas urbanos y sociales para los residentes de esa área, lo que se relacionaría con la creciente malignidad de la segregación residencial en Chile. Por lo tanto, el problema de la segregación residencial en Chile tendría que ver con la relación entre segregación y otros problemas sociales, como el desempleo, drogadicción, embarazo adolescente, deserción escolar, etc.

El análisis de Sabatini, Cáceres y Cerda (2001) para Santiago consiste en usar la desviación estándar del ingreso por hogar como medida de segregación y luego calcular la correlación entre segregación y algunos "problemas" (tiempos de viaje, desempleo, inacción juvenil) en distintas escalas territoriales, con lo cual identifican la escala geográfica para la cual "los efectos de la segregación de hacen más fuertes”. Este análisis genera varios cuestionamientos estadísticos respecto a su validez. El primero es la falta de representatividad de la Encuesta Origen y Destino a distintos niveles geográficos, en particular respecto al ingreso. El segundo es la posibilidad de que exista causalidad inversa y que sean algunos de estos "problemas" los que causen segregación en vez de ser éstos el resultado de la segregación. Si por ejemplo los tiempos de viaje tienen algún grado de relación con los distintos niveles de congestión en la ciudad, lo cual es una externalidad negativa, un modelo económico tipo Tiebout podría explicar perfectamente cómo las distintas valoraciones del tiempo en distintos grupos sociales junto al precio de las viviendas que capitalizan los mejores accesos lleva a segregación residencial (la cual en este caso sería además eficiente). El tercero es la endogeneidad misma de los resultados con la metodología usada, ya que se analizan los datos con el objeto de buscar el efecto negativo mayor hasta que se lo encuentra. El cuarto es que las conclusiones se basan en la correlación muestral sin considerar su error estándar, el cual ni siquiera se reporta. Varias de las correlaciones son 
relativamente bajas y es probable que no se rechace la hipótesis de que estadísticamente son cero o incluso positivas.

Por otro lado, el trabajo de Rodríguez (2001) pone énfasis en la medición de la segregación residencial y en la descripción de este fenómeno para el Área Metropolitana del Gran Santiago (AMGS). El concepto de segregación residencial que utiliza se refiere "al peso que tiene la localización de las unidades sociodemográficas elementales dentro del territorio de referencia en la variación total del atributo entre ellas”, lo que le lleva a utilizar un análisis de varianzas como la medida más adecuada para mirar la relación espacial entre distintos grupos sociales. A partir de variables de segmentación socioeconómica (años de estudio del jefe de hogar, promedio de necesidades básicas insatisfechas (NBI) por hogar, años de estudio población total y años de estudio del grupo 30 a 39 años), calcula el Índice de Disimilitud para Santiago usando los datos de los censos de 1982 y 1992, estimándolos en 0,47 y 0,4 respectivamente.

A partir del análisis espacial de las diferencias entre las comunas de la AMGS de las variables socioeconómicas consideradas, Rodríguez señala que hay "diferencias ostensibles" en las medias comunales y que aquellas comunas que tienen condiciones socioeconómicas superiores están agrupadas todas en un sector de la ciudad, mientras que las que tienen condiciones socioeconómicas más bajas se ubican más bien en la periferia.

La conclusión es que hay atributos que están desigualmente distribuidos entre la población y que las comunas más cercanas al centro de la ciudad se caracterizan por una baja segregación residencial interna, mientras que en la periferia la segregación residencial es mayor. Esto se traduce en que los niveles de segregación difieren entre comunas, al igual que las modalidades de segregación, pero resalta el hecho de que el AMGS está todavía lejos de medidas extremas de segregación como las que alguna vez se han encontrado en ciudades de Estados Unidos para temas de raza. Por último, señala que los niveles de segregación existentes en el Gran Santiago pueden ser mucho mayores que los estimados a partir de variables de educación y de necesidades básicas insatisfechas, por lo que si se estimara con ingresos los niveles de segregación "serían mucho más notorios".

Por último, es importante destacar el trabajo de Vargas (2006), quien más allá de intentar medir la segregación residencial en Chile 
realiza también un análisis estadístico que permita explicar sus causas. A partir de datos de la encuesta Casen de los años 1992 y 2003 utiliza el nivel educacional del jefe de hogar para estimar el Índice de Disimilitud, estimándolo en 0,61 y 0,64 respectivamente, lo que mostraría que la segregación residencial en Santiago ha permanecido constante. Uno de los problemas detrás de este cálculo es que la encuesta Casen, como se discute más adelante, no es representativa a niveles geográficos muy desagregados (y aunque lo fuera, el margen de error es lo suficientemente grande que impide tener estimadores precisos).

Con el objeto de estudiar las causas de la segregación residencial, Vargas (2006) estima regresiones estadísticas que permitan explicar los índices de segregación calculados. Los resultados muestran que los principales determinantes de la segregación en Santiago son el costo de arriendo, el número de personas en el hogar, la propiedad de la vivienda, el estado civil y si el hogar recibió subsidio del Estado para vivienda. Éste es un resultado muy interesante, ya que las fuerzas detrás de la segregación residencial económica en Chile son del mismo tipo que las que explican la pobreza.

Como podemos ver, las distintas miradas sobre segregación residencial en Santiago apuntan hacia la existencia de segregación, si bien no hay consenso respecto a qué tan crítico es su nivel ni tampoco respecto a sus efectos. Tampoco hay consenso sobre las causas de la segregación, que podrían ser desde el endógeno comportamiento de las elites que desean vivir entre semejantes hasta la política social del gobierno, que es exógena a las decisiones de ubicación de muchos hogares. Por último, parte de la evidencia para Santiago tiene varias deficiencias estadísticas que, si bien pueden deberse parcialmente a las limitaciones de los datos existentes, requieren ser discutidas y consideradas para dimensionar en forma correcta los resultados de cada estudio.

Con el objeto de contribuir a la discusión de la segregación residencial en Chile, en las próximas tres secciones se presentan distintos análisis empíricos complementarios sobre la segregación comunal en la Región Metropolitana (RM). Las razones para analizar segregación a nivel comunal, en vez de a nivel de distritos censales o barrios, tienen que ver tanto con el tipo de datos disponibles y su representatividad, lo cual impone algunas limitaciones que se discuten más adelante, como con la institucionalidad de las políticas públicas en Chile. En este 
último aspecto, la provisión de bienes públicos locales se realiza a nivel comunal y las municipalidades juegan un rol importante al respecto. Adicionalmente, muchas de las políticas públicas relacionadas con reducción de la pobreza y que podrían tener impacto en la segregación se implementan a nivel comunal.

\section{Evidencia a partir de los censos (sin ingresos)}

Tal como se ha hecho en algunos estudios mencionados en la sección anterior, es posible obtener una primera mirada a la segregación residencial en la Región Metropolitana a partir de los datos que entregan los censos de población y vivienda.

El Censo de Población y Vivienda entrega información detallada que permite describir la distribución espacial de algunas características relacionadas con la calidad de vida de las personas, en particular, las que tienen que ver con la calidad de las viviendas, el acceso a bienes de consumo y algunas características socioeconómicas. La limitante principal de los censos, sin embargo, es que no tienen datos de ingresos, que es probablemente la dimensión más relevante sobre la cual analizar la segregación residencial en el caso de Chile.

La razón principal para usar los censos, si bien su periodicidad es cada diez años, es su representatividad estadística de áreas geográficas más pequeñas. A diferencia de los censos, las encuestas de hogares no son representativas a nivel comunal ni a nivel de distrito censal y mirar segregación a nivel regional, nivel en el cual las encuestas sí son representativas, resulta demasiado amplio para un fenómeno que se ha estudiado y observado esencialmente a nivel de ciudades.

Esta mirada entonces tiene por objetivo simplemente mostrar qué ha sucedido en el tiempo con las características y propiedad de las viviendas, con los bienes a los que han tenido acceso los hogares y con las características socioeconómicas de los miembros que lo componen, como su nivel de educación y el tipo de ocupación. A partir de los datos de los censos de 1992 y 2002 es posible comparar las condiciones de las comunas de la Región Metropolitana y analizar los cambios en calidad de vida que ha experimentado la población en los diez años que transcurrieron entre los dos censos ${ }^{3}$.

\footnotetext{
${ }^{3}$ Los gráficos que se mencionan en esta sección están en el Anexo.
} 


\section{Características de la vivienda}

Si consideramos los materiales de las paredes, vemos que en todas las comunas ha aumentado la proporción de viviendas con paredes de ladrillo y/o cemento, mientras que ha disminuido la proporción de viviendas con paredes de madera y otros materiales aún más inestables. Los datos muestran que la distribución de los materiales se ha vuelto más igualitaria, en tanto la mayoría de las comunas de la RM tiene sobre un 60 por ciento de viviendas con paredes de material más sólido y duradero, como lo son el ladrillo y el cemento (Gráfico 1A). De igual forma, la mayoría de las comunas tiene menos de $10 \%$ de las viviendas con paredes de adobe y la varianza entre comunas se ha reducido en forma importante entre los dos períodos censales (Gráfico 2A).

Si miramos lo que sucede con otros materiales para las paredes que son altamente inestables y poco sólidos (barro empajado, quincha, pirca y desechos como lata, cartones y plástico), encontramos que la proporción de paredes que tienen este tipo de materiales para la vivienda ha aumentado levemente respecto de 1992. De hecho, la distribución en 1992 estaba fuertemente concentrada entre 0 y 2 por ciento, lo cual sigue siendo cierto para el año 2002 pero con una distribución que se ha extendido (aumentado la varianza). Es así como en 2002 en algunas comunas el uso de estos materiales sobrepasa el 5\%, como Huechuraba y Lo Espejo, lo que ha contribuido a aumentar la desigualdad en la distribución de viviendas con este tipo de materiales para las paredes.

En cuanto al piso de las viviendas, un cambio notable en términos de menor segregación ha ocurrido con las viviendas con piso de tierra (Gráfico 3A). En 1992, si bien apenas un 2,6\% de las viviendas en la RM tenía piso de tierra, hubo varias comunas con porcentajes superiores a $5 \%$ e incluso unas pocas con porcentajes en torno al $20 \%$. En 2002, todas las comunas tienen un porcentaje menor que el $2 \%$. También es relevante el aumento del uso de la alfombra muro a muro que, a diferencia de lo que ocurría en 1992, ya no está sólo en las comunas de más altos ingresos de la RM. Por otro lado, el baldosín, que era más escaso en 1992 —cuando en la mayoría de las comunas menos del 5\% de las viviendas tenía piso de baldosín-, ha conseguido instalarse con mayores niveles de igualdad en gran parte de las comu- 
nas de la RM y en 2002 hay muchas comunas donde más del 20\% de las viviendas tiene baldosín (Gráfico 4A).

En cuanto al techo de las viviendas, el material más utilizado sigue siendo el pizarreño y su distribución no ha cambiado en forma significativa entre 1992 y 2002. Por otro lado, el techo de tejuelas, si bien en los dos períodos se encuentra en mucho mayor proporción en las comunas de más altos ingresos que en el resto de la RM, se hizo más accesible y en el 2002 se encuentra en un mayor número de comunas y en mayor proporción que en 1992 (Gráfico 5A).

Resulta aún más interesante observar lo que ha sucedido con los materiales para el techo de peor calidad, como fonolita, fibra de vidrio o paja embarrada. Aunque la proporción es baja en la RM, los datos muestran que ha habido una importante disminución de la proporción de viviendas con techos de este tipo para todas las comunas y la media ha caído considerablemente. La distribución de este tipo de viviendas se ha concentrado en cerca del 0 por ciento de las viviendas con una varianza mucho menor que en 1992 (Gráfico 6A).

Si bien es difícil sacar muchas conclusiones en lo que concierne a las características de propiedad de la vivienda, ya que reflejan una mezcla de distintas preferencias respecto a la propiedad, y del acceso al crédito que tienen o no los hogares, hay que destacar que los datos comparados muestran que ha aumentado la proporción de familias que han pagado totalmente su vivienda. En 2002 la mayoría de las comunas tenía un 40 a 70 por ciento de familias con viviendas totalmente pagadas. Lo más interesante es que las comunas con mayor proporción de la población con sus viviendas pagadas no son las comunas de mayores ingresos. Estas comunas tampoco son las que tienen mayores proporciones de viviendas pagadas a plazo. De hecho, lo que muestran los datos es que estas comunas recién aparecen en forma significativa cuando se consideran las viviendas en arriendo. Tanto en 1992 como en 2002, las comunas de altos ingresos son las que tienen una mayor proporción de la población arrendando las viviendas que habitan.

Ahora, si consideramos el tipo de vivienda, la mayor parte de la población de la RM vive en casas y eso no cambia mucho entre 1992 y 2002 (Gráfico 7A). Lo que sí cambia es la proporción de la población que vive en departamentos, lo que refleja un importante desarrollo inmobiliario de este tipo de viviendas en el período (Gráfico 8A). Si bien el aumento en la proporción de departamentos se concentró en 
comunas de altos ingresos, ya en 2002 los datos mostraban señales de extenderse con fuerza hacia otras comunas, como Lo Prado, Macul y San Miguel.

Por último, es importante considerar lo que ha sucedido con las viviendas de tipo mediagua, que han sido tradicionalmente la expresión más clara de la desigualdad y pobreza en nuestras ciudades. Este tipo de vivienda ha experimentado el más importante descenso entre 1992 y 2002 (Gráfico 9A). En 1992 había claramente una mayor desigualdad en la distribución de la población que vivía en mediaguas en las comunas de la RM. En 2002 no sólo disminuyó la proporción de la población que vive en mediaguas, sino también ocurrió que esa proporción cayó más en aquellas comunas donde más había mediaguas en 1992, como en Huechuraba, Peñalolén y Cerro Navia.

\section{Acceso a bienes durables en el hogar}

Además de las características de la vivienda, es relevante considerar los bienes durables que tienen los hogares, ya que reflejan el nivel de consumo o ingreso permanente de las familias. En general, los datos respecto a la tenencia de estos bienes muestran que el acceso que las familias tienen a ellos ha aumentado significativamente, en gran parte debido al crecimiento económico y la apertura comercial, reduciéndose o eliminándose la segregación residencial en esta dimensión. Es precisamente en este ámbito que se encuentran mayores reducciones de desigualdad en la población y entre las comunas de la RM en el período entre 1992 y 2002.

Es impresionante lo que ha ocurrido, por ejemplo, con el refrigerador (Gráfico 10A). A principios de la década de 1990, aunque la tenencia de refrigerador era alta, había una gran desigualdad en la proporción de hogares con refrigerador entre las distintas comunas de la RM, desde comunas como Vitacura con casi $100 \%$ hasta comunas como San Pedro con menos de 25\%. En 2002, en cerca del 90 por ciento de las comunas la población que tiene refrigerador en la casa fluctúa entre el 90 y 100 por ciento y en la comuna con menor proporción, Alhué, más de $60 \%$ de la población tiene refrigerador.

En el caso de los televisores, claramente ha desaparecido el televisor en blanco y negro y el televisor en colores está hoy en más del 90\% de los hogares de todas las comunas de la RM, y así se ha 
eliminado la desigualdad que había en el acceso a este bien en 1992, con comunas como Vitacura con casi el $100 \%$ y comunas como San Pedro con menos de 5\% (Gráfico 11A). Incluso el acceso al videograbador aumentó también, pasando de un promedio de menos del 20\% de los hogares en 1992 a más de 45\% de los hogares en 2002. En 1992 había sólo seis comunas donde más del $40 \%$ de los hogares tenía videograbador, mientras que en 2002 había más de 30 comunas con al menos 40\% (Gráfico 12A).

El acceso a lavadora se duplicó en promedio entre 1992 y 2002, desde alrededor de $40 \%$ hasta más de $80 \%$ de los hogares (Gráfico 13A). En 2002 en todas las comunas más del 60\% de los hogares tenía lavadora, con lo que la distribución se hizo más igualitaria entre comunas, disminuyendo en forma significativa la brecha entre las comunas con menor y mayor proporción de hogares con lavadora.

La tenencia de horno microondas en el hogar también experimentó un salto significativo en este período (Gráfico 14A). En 1992, la inmensa mayoría de los hogares no tenía microondas y en sólo cinco comunas de la RM, las de más altos ingresos, más del 20\% de los hogares lo tenía. En 2002 casi 40\% de los hogares tenía uno y en la gran mayoría de las comunas más del 20\% de los hogares tiene microondas. Éste es un caso en el cual la media de la distribución aumentó en forma significativa, reflejando un mayor acceso al bien, pero aunque la inmensa mayoría de los hogares está mejor también la desigualdad entre comunas aumentó.

Más sorprendente todavía es lo que ha sucedido con los teléfonos celulares. En 1992 éste era un bien sólo accesible para la población de las siete comunas de más altos ingresos, aunque en una proporción bastante baja; en el 2002 el celular ya estaba masificándose, y entre el 40 y 60 por ciento de la población de las comunas de la RM tenía un celular en casa (Gráfico 15A). Es importante resaltar en este caso que, si bien no hay duda de que en términos de acceso los hogares y todas las comunas estaban mejor en 2002 que en 1992, la distribución es más desigual en 2002 (al igual que en el caso de acceso al microondas). La razón es bastante obvia: la variación entre comunas en 1992 es muy baja porque en la gran mayoría de las comunas nadie tenía celular y en ese sentido era más igualitaria.

De igual forma el acceso al teléfono fijo aumentó considerablemente en el período. En 1992 alrededor del 25\% de los hogares tenía 
teléfono fijo y en la mayoría de las comunas menos de un 40\% de los hogares tenía uno. En el año 2002, el 60\% de los hogares de la RM tenía teléfono fijo y en casi todas las comunas más de un $40 \%$ de su población contaba con teléfono fijo en el hogar (Gráfico 16A).

\section{Características socioeconómicas del hogar}

Como hemos visto en los datos anteriores, tanto la calidad de las viviendas como el acceso a bienes de consumo durables no sólo han aumentado significativamente entre 1992 y 2002 sino que además la variación entre comunas se ha reducido en general, reflejando una menor desigualdad y una menor segregación en términos de varianza intercomunal. Sin duda, ambos aspectos están correlacionados con el ingreso per cápita de los hogares, pero en forma complementaria es importante también analizar qué ha sucedido con algunas características socioeconómicas que están correlacionadas con el capital humano del jefe de hogar, tal como suele mirarse en mediciones de segregación.

Si consideramos la educación del jefe de hogar los datos revelan que la distribución de la proporción de jefes de hogar por comuna que sólo tienen educación básica completa es similar entre 1992 y 2002 (Gráfico 17A). Esta proporción disminuyó en casi 10 puntos porcentuales en promedio durante el período y cayó en algún grado en todas las comunas. La diferencia mayor entre comunas sigue siendo respecto a las seis comunas de mayores ingresos, las cuales tienen una fracción muy pequeña de jefes de hogar cuyo máximo nivel educacional alcanzado es la educación básica. Por otro lado, la proporción de jefes de hogar cuyo nivel educacional es educación media aumentó levemente en casi todas las comunas y la varianza de la distribución disminuyó, reflejando una mayor homogeneidad entre comunas que en 1992 (Gráfico 18A).

Es interesante lo que ha sucedido con los jefes de hogar con educación superior y, en particular, lo que ha sucedido con la formación técnica. La proporción de jefes de hogar con educación técnica ha aumentado significativamente en todas las comunas, pasando de un promedio de $2,5 \%$ a casi $8 \%$, pero proporcionalmente ha aumentado más en varias de las comunas que tenían menor proporción de jefes de 
hogar con educación técnica en 1992, como Lampa, Padre Hurtado, La Pintana, Cerro Navia y Huechuraba (Gráfico 19A).

Finalmente, en cuanto al nivel de educación máximo alcanzado, la fracción de jefes de hogar con educación universitaria completa aumentó levemente entre 1992 y 2002 y la distribución es muy similar en ambos períodos (Gráfico 20A). Claramente es en esta dimensión donde se observan mayores diferencias entre las distintas comunas de la RM. Si bien la distancia se ha reducido un poco, las comunas asociadas a hogares de mayores ingresos se distancian significativamente de todas las restantes. Probablemente, esta distancia debiera disminuir en forma importante durante los próximos años, ya que la educación universitaria ha aumentado mucho su matrícula y el número de alumnos que son la primera generación familiar en ir a la universidad ha crecido también.

En relación al empleo, si bien los momentos del ciclo económico juegan un rol importante y pueden ser distintos en ambos períodos, los datos muestran que la proporción de jefes de hogar que se encontraba trabajando casi no cambió entre 1992 y 2002. Lo que sí ha cambiado en forma significativa es la proporción de mujeres que trabajan, la cual ha subido en todas las comunas, con la excepción de las comunas de altos ingresos, donde se ha mantenido relativamente igual o incluso ha disminuido (Gráfico 21A), reduciéndose la varianza entre comunas. En 1992 la mayoría de las comunas tenía un 20 a 30 por ciento de mujeres trabajando, mientras que en 2002 la proporción estuvo entre 30 y 40 por ciento. Este cambio puede tener un impacto significativo en el nivel de ingreso per cápita del hogar que disminuye la desigualdad de ingresos entre comunas.

La Tabla $\mathrm{N}^{\mathrm{o}} 1$ presenta los índices de Disimilitud y Exposición de algunas de las características de vivienda, tenencia de bienes y características socioeconómicas que se han analizado en esta sección. Más allá de que en general el valor de los índices no es particularmente alto, en la mayoría de los casos los niveles de segregación han disminuido entre 1992 y 2002.

La evidencia empírica que nos entrega el análisis de los censos de 1992 y 2002, tal como se ha mostrado en esta sección, refleja una realidad bastante más positiva de lo que tradicionalmente se cree y se ha señalado en los análisis sobre segregación. La calidad de las viviendas y el acceso a bienes han mejorado mucho entre 1992 y 2002, pero 


\begin{tabular}{|c|c|c|c|c|c|c|}
\hline & & & \multicolumn{2}{|c|}{ Disimilitud } & \multicolumn{2}{|c|}{ Exposición } \\
\hline & & & 1992 & 2002 & 1992 & 2002 \\
\hline \multirow[t]{4}{*}{ Características de la vivienda } & Pared & internit y desechos & 0,24 & 0,30 & 0,01 & 0,04 \\
\hline & Piso & tierra & 0,30 & 0,27 & 0,04 & 0,01 \\
\hline & Techos & fibra de vidrio, & & & & \\
\hline & & $\begin{array}{l}\text { fonolita, paja y } \\
\text { desechos }\end{array}$ & 0,28 & 0,24 & 0,03 & 0,01 \\
\hline \multirow[t]{8}{*}{ Bienes durables en el hogar } & Televisor & no tiene & & & & \\
\hline & colores & & 0,23 & 0,27 & 0,71 & 0,15 \\
\hline & Refrigerador & no tiene & 0,25 & 0,25 & 0,61 & 0,29 \\
\hline & Celular & no tiene & 0,50 & 0,19 & 152,85 & 1,10 \\
\hline & $\begin{array}{l}\text { Mini- } \\
\text { componente }\end{array}$ & no tiene & 0,17 & 0,14 & 1,94 & 0,38 \\
\hline & Lavadora & no tiene & 0,16 & 0,15 & 1,54 & 0,22 \\
\hline & Microondas & no tiene & 0,54 & 0,23 & 46,73 & 1,77 \\
\hline & Videograbador & no tiene & 0,29 & 0,16 & 4,91 & 1,30 \\
\hline \multirow{2}{*}{$\begin{array}{l}\text { Características socioeconómicas } \\
\text { del hogar }\end{array}$} & Educación & sin educación & 0,22 & 0,26 & 0,05 & 0,03 \\
\hline & Empleo & desempleado & 0,08 & 0,09 & 0,07 & 1,84 \\
\hline
\end{tabular}

adicionalmente las diferencias entre comunas se han reducido en forma significativa.

En la dimensión de nivel educacional la evidencia es menos positiva en términos de cambios significativos en el tiempo en el nivel educacional promedio de la población. Adicionalmente, los datos muestran que las diferencias entre comunas son importantes en algunos casos, en particular en educación básica y en educación universitaria, donde unas pocas comunas concentran una fracción importante de personas con alto nivel educacional y muy pocas personas con bajo nivel educacional.

\section{Segregación comunal en la Región Metropolitana (con ingresos)}

Tal como se mencionó en la sección anterior, la segregación residencial por ingresos es una de las dimensiones más relevantes de analizar en la RM. Para ello se requiere contar con datos de ingresos 
que sean representativos a un nivel geográfico relativamente desagregado (a nivel comunal por lo menos). La principal dificultad radica entonces en que los datos censales, que son representativos a niveles muy desagregados, no contienen ingreso y los datos de las encuesta de hogares (Casen por ejemplo), que contienen datos de ingreso, no son representativos a niveles desagregados ${ }^{4}$.

La solución a este problema se encuentra en el uso de metodologías que permiten combinar los datos de encuestas con los datos censales para obtener estimadores geográficos desagregados de algunos indicadores de bienestar asociados a ingreso (Elbers, Lanjouw y Lanjouw, 2003). Para esto lo primero que se hace es estimar un modelo de ingreso por hogar utilizando características observables que están presentes en ambas bases (encuesta de hogares y censo). Posteriormente, con los parámetros estimados se estima el nivel esperado de los indicadores de desigualdad y pobreza con los datos que se tienen en el censo, con lo cual se obtiene un estimador consistente para estos indicadores junto a su error estándar asociado (una explicación completa de la metodología para quien se interese en los detalles se encuentran en el Anexo Metodológico).

Para el caso de Chile, los trabajos de Agostini y Brown (2007) y Agostini, Brown y Góngora (2008) utilizan este tipo de metodologías para generar indicadores de pobreza y desigualdad a nivel comunal. Replicando dicho análisis para la Región Metropolitana, se presentan a continuación los resultados para el ingreso per cápita del hogar, la tasa de pobreza y el coeficiente de Gini para las comunas de la RM, lo que permite analizar segregación residencial en esas dimensiones.

\section{Ingreso per cápita del hogar}

El Gráfico No 1 muestra la distribución del ingreso per cápita del hogar promedio de las comunas de la RM. Las comunas con menor ingreso per cápita del hogar son Alhué y La Pintana, con alrededor de \$ 84 mil y \$ 88 mil respectivamente. Por otro lado, las comunas de

${ }^{4}$ Incluso aunque fueran representativos, el error estándar es tan grande que los intervalos de confianza para cada indicador no son muy informativos. Por ejemplo, la tasa de pobreza en La Pintana se estima entre 25,3\% y 45,7\% utilizando los datos de la Casen 2003. 
GRÁFICO No 1: $\quad$ INGRESO PER CÁPITA DEL HOGAR POR COMUNA EN LA RM 2003

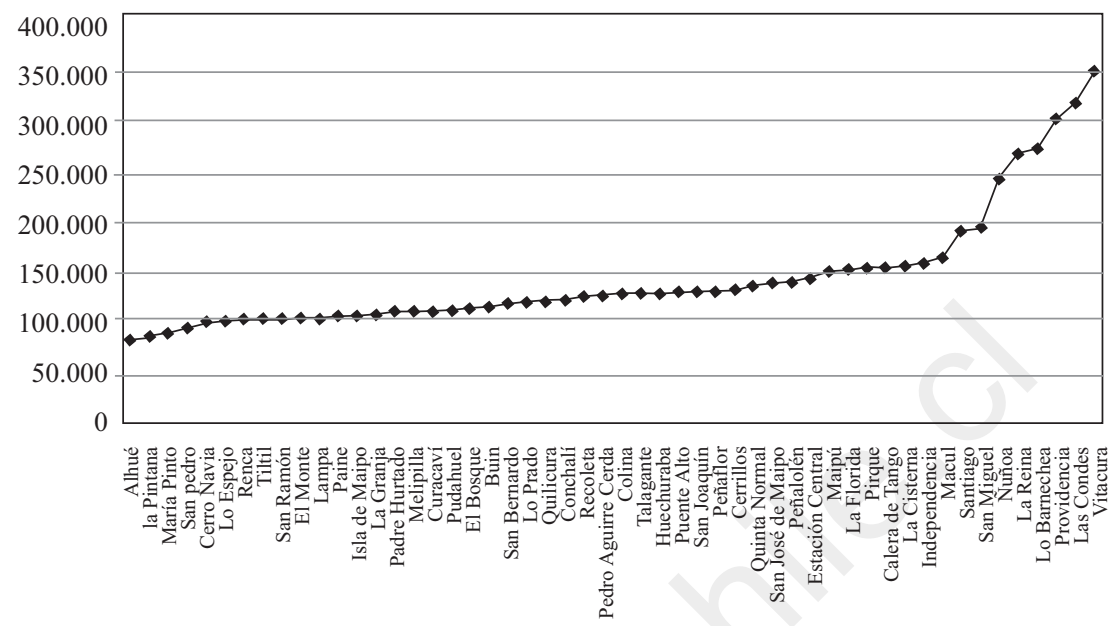

más alto ingreso son Vitacura y Las Condes con \$ 352 mil y \$ 320 mil respectivamente.

La distribución de coeficientes Gini es relativamente uniforme hasta llegar a las seis comunas de ingresos más altos en la RM, donde ocurre un salto significativo respecto al resto de las comunas. Si bien esto refleja que los hogares de más altos ingresos tienden a concentrarse en unas pocas comunas, lo cual puede ser el resultado de un mecanismo de autoselección por preferencias respecto a bienes públicos locales (conocido como equilibrio de Tiebout en la literatura económica), en todo el resto de la RM los niveles de segregación en términos de ingreso son bastante bajos.

\section{Pobreza}

El Gráfico No 2 muestra la distribución de la tasa de pobreza comunal en la RM.

Las comunas con mayor tasa de pobreza en la RM son Cerro Navia y La Pintana, con 30\% y 35\% respectivamente. Las comunas con menores tasas de pobreza urbana en la Región Metropolitana son Vitacura, Providencia y Las Condes, con tasas de pobreza de 1,4\%, 2,5\% y 3,4\% respectivamente. 
GRÁFICO N 2: $\quad$ POBREZA COMUNAL EN LA RM 2003 (\% HOGARES POBRES)

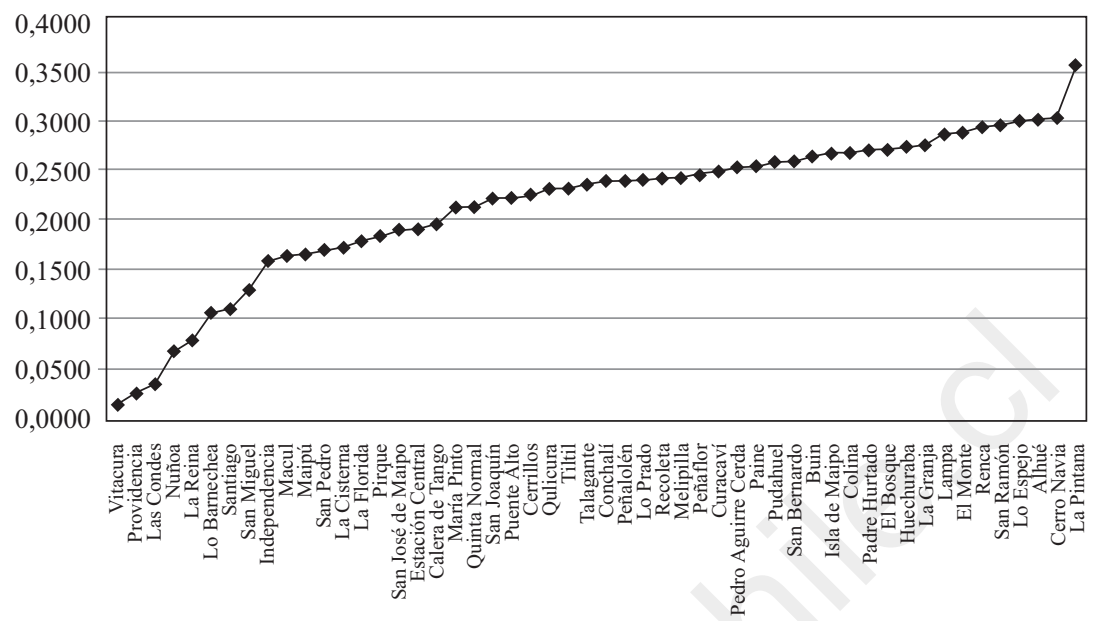

Si para efectos de calcular los índices de segregación residencial más utilizados separamos a la población entre pobres y no pobres, el Índice de Disimilitud es de 0,21 y el Índice de Exposición es de 0,34. Estos valores no son especialmente altos, si bien es cierto que los niveles de pobreza existentes en cinco comunas son particularmente bajos respecto al promedio de la RM.

\section{Desigualdad}

El Gráfico No 3 muestra la distribución del coeficiente de Gini comunal en la RM. Las comunas con mayor desigualdad son Calera de Tango y Colina con Ginis de 0,54 y 0,53, respectivamente. Las comunas con menor desigualdad son Vitacura y Providencia con Ginis de 0,43 y 0,44, respectivamente. En este caso la distribución es bastante plana y no hay comunas particularmente desiguales o particularmente iguales, si bien el valor del coeficiente de Gini es relativamente alto para casi todas las comunas comparado con el de países desarrollados. Si la segregación residencial por ingresos fuera muy alta en la RM, los coeficientes de Gini de cada comuna debieran ser relativamente bajos, ya que en el extremo habría comunas sólo con pobres y comunas sólo con ricos, con lo que la desigualdad comunal sería muy baja. 
GRÁFICO N ${ }^{\circ}$ 3: $\quad$ DESIGUALDAD COMUNAL EN LA RM 2003 (COEFICIENTE DE GINI)

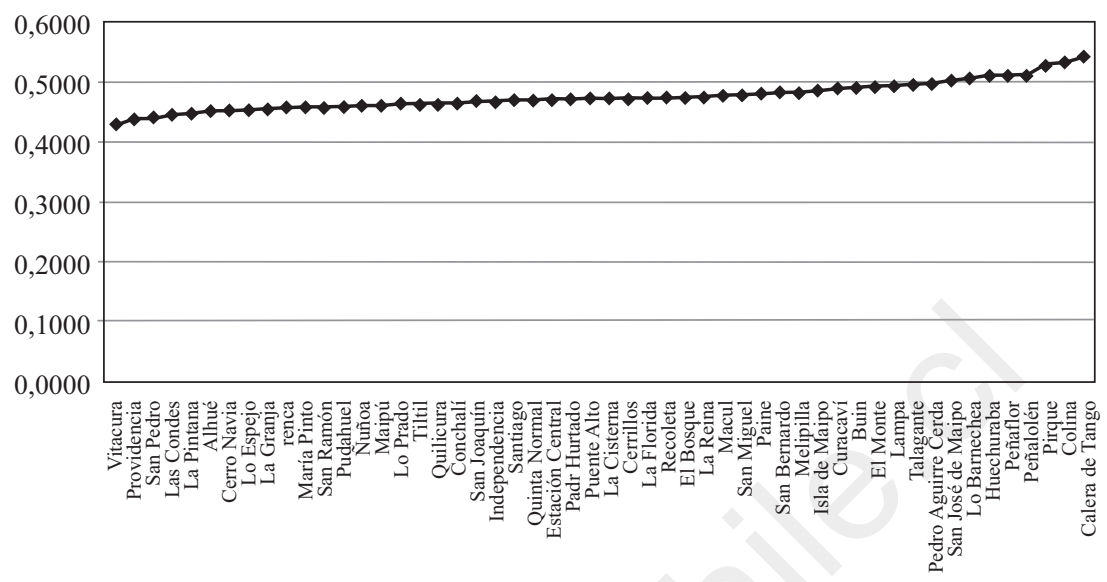

Un aspecto interesante de considerar es la relación que existe entre la pobreza y la desigualdad en las comunas. Una de las cosas que se han dicho sobre segregación en Santiago es que las comunas más pobres son heterogéneas, porque en ellas conviven familias pobres y de clase media, mientras que las comunas más ricas son homogéneas. El Gráfico $N^{\circ} 4$ muestra el porcentaje de pobres y el coeficiente de Gini para cada comuna en la RM. Tal como se observa, las comunas menos desiguales son, en general, las que tienen los menores (La Pintana, Cerro Navia y Lo Espejo) y los mayores (Vitacura, Providencia y Las Condes) porcentajes de pobreza. No es claro entonces que exista una relación lineal entre pobreza y desigualdad ni tampoco se ratifica claramente en los datos que las comunas más pobres sean más heterogéneas que las comunas de altos ingresos. De hecho, La Pintana y Las Condes tienen prácticamente el mismo nivel de desigualdad.

De igual forma, se ha dicho que las comunas en el centro de Santiago son más homogéneas y menos segregadas que las comunas de la periferia de la ciudad. Sin embargo, esto no se refleja en los datos de desigualdad en los ingresos. Es así como por ejemplo Quilicura, en la periferia, tiene un Gini equivalente al de Independencia y Santiago, en el centro; Puente Alto, en la periferia, tiene un Gini similar al de Estación Central y Quinta Normal, ambas céntricas; y San Bernardo, en las afueras de la ciudad, tiene un Gini igual al de San Miguel, muy cerca del centro. 
GRÁFICO N 4: RELACIÓN ENTRE POBREZA Y DESIGUALDAD COMUNAL EN LA RM 2003

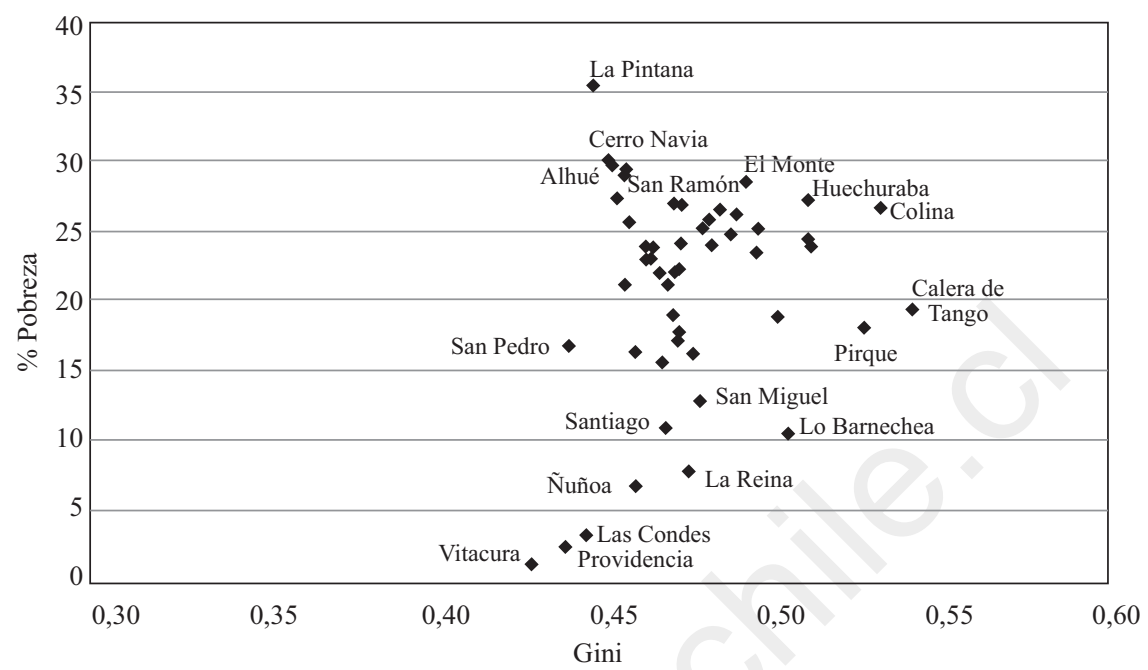

\section{Un ejercicio interesante: Reasignación aleatoria de comunas}

El análisis de los datos censales de 1992 y 2002 mostró avances importantes en la calidad de vida de las familias en ese período, tanto en las características de las viviendas como en el acceso a bienes durables y niveles de educación, y junto a ello se observa también una disminución de las brechas entre comunas. Sin embargo, hay diferencias que persisten en el acceso a unos pocos bienes durables, algunos materiales de techo, paredes y piso de las viviendas y, en particular, en que el jefe de hogar tenga un nivel de educación superior completa. Una parte importante de estas desigualdades entre comunas de la RM que persisten en el período entre censos se explica mayoritariamente por tres comunas que en varias dimensiones se alejan del resto: Vitacura, Las Condes y Providencia. Adicionalmente, son las comunas que aparecen como más homogéneas en su composición al tener los menores índices de desigualdad de la RM.

Si bien es imposible hacerlo en el mundo real, una pregunta interesante de responder es qué pasaría si suprimimos esas tres comunas de la RM y reasignamos en forma aleatoria a sus habitantes en el resto de las comunas. Si efectivamente la segregación residencial se debe a que hay tres comunas donde se concentran las personas de más 
altos ingresos, más educadas, con mejores viviendas y mayor acceso a bienes durables, entonces esta reasignación debiera tener un impacto importante en reducir la segregación en la $\mathrm{RM}^{5}$.

El proceso de reasignación aleatoria es bastante simple y consiste en asignar a cada hogar de Vitacura, Las Condes y Providencia un nuevo código comunal elegido aleatoriamente para luego volver a generar los mismo gráficos e índices ya analizados. El ejercicio se realizó con los datos del Censo 2002.

Los resultados de este ejercicio muestran que, en general, las distribuciones no cambian mucho en el caso de muchas características de la vivienda, la propiedad de activos durables y la situación de empleo del jefe de hogar. En particular, se mantienen casi inalteradas las distribuciones comunales de paredes de adobe y de desechos; techos de pizarreño, fonolita, fibra de vidrio y paja embarrada; pisos de parquet y entablado; viviendas de tipo casa y mediaguas; y viviendas completamente pagadas. Lo mismo ocurre con las distribuciones de tenencia de refrigerador, televisor en colores, minicomponente, lavadora y celular.

Hay algunas distribuciones que mejoran levemente, en términos de hacerse más uniformes y disminuir su varianza, como resultado de la reasignación aleatoria. En el caso de las características de construcción de las viviendas, junto a la disminución en la varianza en todas las comunas aumenta la proporción de viviendas con techo de tejuelas y pisos con alfombra muro a muro y disminuye la fracción que tiene piso de tierra. En cuanto a la propiedad y tipo de viviendas aumenta la fracción de viviendas arrendadas y disminuye fuertemente la proporción de departamentos en las comunas, reduciéndose la varianza intercomunal. En el caso de los bienes que tienen los hogares, se reduce fuertemente la varianza en las distribuciones de videograbador y microondas, aumentando la media y haciéndose más uniforme la distribución. En el caso de características socioeconómicas, esto ocurre con la distribución de la participación laboral femenina.

Los mayores cambios como resultado de este ejercicio de reasignación aleatoria de hogares ocurren en la distribución entre comunas

${ }^{5}$ Las conclusiones de este ejercicio están limitadas por el hecho de ser un ejercicio estático que no considera la posterior movilidad de los hogares que son reasignados a otra comuna. Sin embargo, los resultados permiten contrastar algunas creencias respecto al grado de segregación que existiría en Santiago y el rol que juegan las comunas más ricas del "sector oriente". 
de algunas características socioeconómicas de las personas al interior del hogar, especialmente las relacionadas con nivel educacional. En todas las comunas la fracción de jefes de hogar sólo con educación básica completa disminuye y aumenta la de los que tienen educación superior, haciéndose más homogénea la distribución entre comunas en ambos casos.

La Tabla № 2 muestra los índices de Disimilitud y Exposición para las mismas variables de la Tabla $\mathrm{N}^{0} 1$ presentada anteriormente, pero esta vez comparando los datos del Censo 2002 antes y después de la reasignación aleatoria. Los cambios en los niveles de segregación no son de magnitud significativa en la mayoría de los casos y adicionalmente ocurren en ambas direcciones, no sólo disminuye la segregación sino que en algunas dimensiones aumenta.

Los resultados de este ejercicio confirman la evidencia intercensal presentada anteriormente, en el sentido de que no hay niveles de desigualdad ni segregación importantes entre comunas para la mayoría de las características de las viviendas y los bienes durables que tienen

TABLA N ${ }^{\circ}$ 2: $\quad$ ÍNDICES DE SEGREGACIÓN CON DATOS CENSALES Y REASIGNACIÓN ALEATORIA

\begin{tabular}{|c|c|c|c|c|c|c|}
\hline \multirow[b]{2}{*}{ Características de la vivienda } & \multirow[b]{2}{*}{ Pared } & & \multicolumn{2}{|c|}{ Disimilitud } & \multicolumn{2}{|c|}{ Exposición } \\
\hline & & internit y desechos & s 0,30 & 0,23 & 0,04 & 0,50 \\
\hline & Piso & tierra & 0,27 & 0,23 & 0,01 & 0,02 \\
\hline & Techos & fibra de vidrio, & & & & \\
\hline & & $\begin{array}{l}\text { fonolita, paja y } \\
\text { desechos }\end{array}$ & 0,24 & 0,21 & 0,01 & 0,01 \\
\hline \multirow[t]{8}{*}{ Bienes durables en el hogar } & Televisor & no tiene & & & & \\
\hline & en colores & & 0,27 & 0,16 & 0,15 & 0,14 \\
\hline & Refrigerador & no tiene & 0,25 & 0,21 & 0,29 & 0,22 \\
\hline & Celular & no tiene & 0,19 & 0,31 & 1,10 & 2,39 \\
\hline & $\begin{array}{l}\text { Mini- } \\
\text { componente }\end{array}$ & no tiene & 0,14 & 0,11 & 0,38 & 0,36 \\
\hline & Lavadora & no tiene & 0,15 & 0,13 & 0,22 & 0,21 \\
\hline & Microondas & no tiene & 0,23 & 0,15 & 1,77 & 1,52 \\
\hline & Videograbador & no tiene & 0,16 & 0,11 & 1,30 & 1,20 \\
\hline \multirow{2}{*}{$\begin{array}{l}\text { Características socioeconómicas } \\
\text { del hogar }\end{array}$} & Educación & sin educación & 0,26 & 0,22 & 0,03 & 0,03 \\
\hline & Empleo & desempleado & 0,09 & 0,08 & 1,84 & 2,66 \\
\hline
\end{tabular}


los hogares. En cambio sí existen diferencias importantes en los niveles de educación de los jefes de hogar y hay un grado de concentración relativamente importante de los que tienen educación superior completa en unas pocas comunas del sector oriente de la ciudad.

\section{Conclusiones}

En general, hay bastante acuerdo respecto a los niveles y la evolución que han tenido tanto la pobreza como la desigualdad en Chile y en la Región Metropolitana. Sin embargo, no existe igual acuerdo respecto a la segregación. Hay distintas formas de definirla y medirla y las mediciones no son consistentes a lo largo del tiempo, así que tampoco hay acuerdo en su evolución en el tiempo. A pesar de ello, sí existen muchas percepciones y opiniones muy marcadas. La visión dominante respecto a la ciudad de Santiago es que es una ciudad muy segregada, lo cual tendría efectos negativos en la calidad de vida de las personas y el funcionamiento de la ciudad.

Esta percepción negativa de la segregación en Santiago no siempre está bien respaldada en un análisis empírico riguroso ni se consideran las limitaciones estadísticas que tienen los datos utilizados para proveer evidencia de segregación. Como resultado de estas percepciones muchas veces se sacan más conclusiones que las que los datos realmente permiten. Esto es particularmente cierto dado que no existen datos disponibles de ingresos que tengan representatividad estadística a niveles geográficos más pequeños que una región o algunas comunas muy grandes.

En el análisis empírico se utilizan datos de los censos de 1992 y 2002 y una metodología que permite combinar los datos de la encuesta Casen con los del Censo para generar datos de ingreso representativos a nivel comunal, con el objeto de contribuir a la discusión sobre segregación en Santiago a partir de los datos objetivos y considerando el rol de los ingresos, la pobreza y la desigualdad.

La evidencia empírica que entregan los datos censales es bastante menos dramática que la percepción de la segregación en Santiago. Particularmente, los datos muestran que la calidad de vida, en términos de vivienda y bienes durables, ha mejorado en forma importante durante la última década y las desigualdades entre comunas también se han 
reducido en forma importante. En ese sentido la ciudad se ha vuelto menos segregada, lo que se confirma al calcular los índices de segregación más utilizados en la literatura.

Adicionalmente, algunas afirmaciones sobre Santiago no se ven confirmadas en los datos. Por ejemplo, la idea de que en Santiago las comunas más pobres son heterogéneas, porque en ellas conviven familias pobres y de clase media, mientras que las comunas más ricas son homogéneas no se refleja en los datos de pobreza y desigualdad comunal, ya que tanto las comunas más pobres como las más ricas son las más homogéneas de todas en términos de ingreso per cápita. De igual forma, la percepción de que las comunas en el centro de Santiago son más homogéneas y menos segregadas que las comunas de la periferia de la ciudad tampoco se confirma en los datos de desigualdad en los ingresos y hay comunas periféricas tan heterogéneas u homogéneas en términos de ingreso como las comunas ubicadas en el centro de la ciudad.

La evidencia presentada en este trabajo, sin embargo, es menos positiva en términos de cambios significativos en el tiempo en el nivel educacional promedio de la población. En este sentido, los datos muestran que las diferencias entre comunas son importantes en algunos casos, en particular en educación básica y en educación universitaria, donde unas pocas comunas concentran una fracción importante de personas con alto nivel educacional y muy pocas personas con bajo nivel educacional.

Por último, es importante destacar que en muchos de los indicadores presentados de calidad de vivienda, acceso a bienes durables e ingresos per cápita hay tres comunas que tienden a estar muy arriba del promedio: Vitacura, Providencia y Las Condes. Por un lado, si aplicamos la definición de segregación, esto nos lleva a concluir que son las tres comunas de más altos ingresos las que están segregadas en la ciudad. En este sentido si la preocupación es la segregación per se, la discusión pública debiera centrarse en los hogares de más altos ingresos que son los que efectivamente están segregados del resto de la ciudad donde viven la clase media y los pobres que son la gran mayoría. Por otro lado, esto genera la pregunta de qué pasaría entonces si eliminamos dicha segregación e integramos a todos los hogares de esas tres comunas al resto de la ciudad. Si bien es un ejercicio artificial, los 
resultados de repartir a todas las familias de Vitacura, Las Condes y Providencia en forma aleatoria en el resto de la ciudad muestran que no cambian mucho las cosas. Con pocas excepciones, las distribuciones de las características de la vivienda y de los distintos bienes que poseen los hogares se mantienen casi inalteradas. El único cambio significativo ocurre en la distribución del nivel educacional del jefe de hogar, donde aumenta la proporción que tiene educación superior en todas las comunas y se hace más homogénea la distribución (menor desigualdad entre comunas).

Este último ejercicio no sólo confirma que la calidad de vida ha mejorado mucho y las diferencias entre comunas en la Región Metropolitana se han reducido en forma significativa, sino que la pobreza y las diferencias en capital humano son problemas de mayor magnitud y, por lo mismo, debieran tener mayor prioridad en el debate público que la segregación.

\section{ANEXO METODOLÓGICO}

La metodología general para generar mapas de pobreza y desigualdad geográficamente desagregados consiste en usar los datos de las encuestas de hogares para imputar los datos faltantes de ingreso en el censo y, posteriormente, con las medidas imputadas de ingreso a nivel de hogar, estimar los indicadores de pobreza a niveles más desagregados que los que permiten las encuestas de hogares. Para esto se procede en dos etapas. En la primera etapa se estima un modelo de ingreso por hogar usando los datos de las encuestas pero restringiendo las variables explicativas a las que están contenidas en las dos bases de datos (encuestas de hogares y censo). En la segunda etapa se estima el nivel esperado de los indicadores de pobreza y desigualdad dadas las características observables de la población en el censo.

En términos estadísticos, la metodología consiste en estimar la distribución conjunta del ingreso y un vector de variables que co-varían con el ingreso. Restringiendo el set de variables explicativas a las existentes para personas y hogares en el censo, la distribución conjunta estimada puede usarse para generar la distribución del ingreso para 
cualquier subconjunto de la población en el censo, condicional a las características observadas de ese subconjunto de la población. De esta forma es posible obtener la distribución condicional, la estimación punto y el error estándar de los indicadores de pobreza y desigualdad.

En la primera etapa se estima un modelo que relaciona el ingreso per cápita del hogar $h\left(Y_{h}\right)$, asociado a un cluster geográfico $c$, con un conjunto de características observables $\left(X_{h}\right)$ :

$$
\ln Y_{h c}=E\left[\ln Y_{h c} / X_{h c}\right]+u_{h c}=X_{h c} \beta+\mu_{h c}
$$

El vector de errores $u$ se asume que tiene una distribución con media cero y una matriz varianza-covarianza $\Sigma$ : $f(0, \Sigma)$. Para permitir algún grado de correlación dentro de cada cluster $^{6}$ se utiliza la siguiente especificación:

$$
u_{h c}=\eta_{c}+\varepsilon_{h c}
$$

donde $\eta$, el componente de cluster, y el error idiosincrático son independientes entre sí y no están correlacionados con las variables observables $X_{h c}$.

En general, a la varianza del componente idiosincrático del error $\eta_{\varepsilon}^{2}$ se le puede dar una forma funcional bastante flexible. Con estimadores consistentes de $\beta$, los residuos de la descomposición del error estimado $\hat{u}_{h c}=\hat{u}_{. c}+\left(\hat{u}_{h c}-\hat{u}_{. c}\right)=\eta_{c}^{\wedge}+\hat{\varepsilon}_{h c}{ }^{7}$ pueden usarse para estimar la varianza de $\varepsilon$. La forma funcional que comúnmente se usa para estos efectos es la logística que, entre otras cosas, garantiza tener valores no negativos para la varianza:

$$
\sigma_{\varepsilon}^{2}=\left[\frac{A \hat{\varepsilon}^{z_{h c}^{T} \alpha}+B}{1+\hat{\varepsilon}^{z_{h c}^{T} \alpha}}\right]
$$

Los límites superior e inferior, A y B, se estiman junto al parámetro $\alpha$ usando un procedimiento estándar de pseudo máxima verosi-

\footnotetext{
${ }^{6}$ Correlación entre hogares en la misma área geográfica.

${ }^{7}$ El subíndice “.” en la ecuación representa el promedio sobre dicho índice.
} 
militud. La ventaja de esta forma funcional es que además impide valores excesivamente altos para las varianzas predichas ${ }^{8}$.

Este modelo se estima con los datos de las encuestas de hogares usando una aproximación lineal de la expectativa condicional, permitiendo los efectos geográficos (cluster) y de heterocedasticidad mencionados en la distribución del término de error ${ }^{9}$. Como resultado de la estimación en la primera etapa se obtiene un conjunto de parámetros estimados del modelo: el vector $\beta$, la matriz varianza-covarianza asociada a este vector y los parámetros que describen la distribución de los errores.

En la segunda etapa se utiliza el set de parámetros obtenidos en la primera etapa junto a las características de los hogares en los datos del censo para calcular los indicadores de pobreza y desigualdad por áreas geográficas desagregadas. Específicamente, se combinan los parámetros estimados en la primera etapa con las características observables de cada individuo u hogar en el censo para generar valores predichos del logaritmo del ingreso y los términos de error. Para estos efectos, se utiliza el método de bootstrap que simula los valores del ingreso de cada hogar o de cada individuo. Los valores simulados se basan en la predicción de los ingresos a partir de las características observables $\left(X_{h c}\right)$, los parámetros asociados a esas características $(\beta)$ y los términos de error del cluster $(\eta)$ e idiosincrático del hogar $(\varepsilon)$ :

$$
\hat{Y}_{h c}=\exp \left(X_{h c} \hat{\beta}+\hat{\eta}_{c}+\hat{\varepsilon}_{h c}\right)
$$

donde, para cada hogar, los dos componentes del término de error se obtienen de la distribución empírica descrita por los parámetros en la primera etapa ${ }^{10}$. De igual forma, los coeficientes $\beta$ se obtienen de la

${ }^{8}$ Como se muestra en Elbers et al. (2003), la estimación de los límites A y B sin restricciones genera una distribución bien comportada de residuos predichos. Alternativamente, es posible imponer un límite $\mathrm{B}=0$ y $\mathrm{A}=1,05 \max \left\{\varepsilon_{h c}^{\wedge}\right\}$ y obtener resultados empíricos similares.

${ }^{9}$ Se debe tener en cuenta que los efectos geográficos residuales pueden reducir en una magnitud importante la precisión de los resultados en la segunda etapa. Por esta razón, es de vital importancia explicar la variación en el ingreso debida a la ubicación geográfica, con la elección adecuada de las variables observables. La evidencia empírica muestra que puede ser útil incluir entre las variables explicativas las medias geográficas de algunas variables calculadas con los datos del censo.

${ }^{10}$ Para los dos componentes del error, hogar y cluster se utiliza una distribución normal. Adicionalmente se realizaron estimaciones con una distribución t y una distribución no paramétrica. Sin embargo, al comparar las tasas de pobreza nacional y regionales estimadas con las de la encuesta Casen, la predicción más precisa se obtuvo con una distribución normal multivariada. 
distribución descrita por los estimadores $\beta$ de en la primera etapa y la matriz de varianza y covarianzas asociada a ellos ${ }^{11}$.

Por último, para generar los indicadores de pobreza y desigualdad, se utiliza el set completo de valores simulados de $\hat{Y}_{h c}$ para calcular el valor esperado de dichos indicadores por zona geográfica. El procedimiento se repite $\mathrm{n}$ veces y se obtiene un nuevo set de coeficientes $\beta$ y errores para cada simulación. Para cada subgrupo geográfico se calcula la media y la desviación estándar de los indicadores de pobreza y desigualdad sobre el total de simulaciones. Para una ubicación geográfica dada, estas medias constituyen las estimaciones punto del indicador y las desviaciones estándar corresponden a los errores estándar de estos estimadores.

Llamemos al indicador de pobreza o desigualdad $H$, que es una función $X_{c}, \beta u$ donde $\eta_{c}$ es un vector $N_{c}$ del número de miembros del hogar en la comuna $c$ y $X_{c}$ es un $N_{c} x k$ vector de características observables de los individuos y $u_{c}$ es un $N_{c}$ vector de errores. En esta etapa se estima el valor esperado de $H$, dadas las características de los individuos y hogares que son observadas en el censo y el modelo estimado en la primera etapa:

$$
H_{c}^{E}=E[H / \eta, X ; \xi]
$$

donde $\xi$ es el vector de parámetros del modelo e incluye los parámetros que describen la distribución del error. Reemplazando el vector $\xi$ por un estimador consistente se obtiene:

$$
\hat{H}_{c}^{E}=E[H / \eta, \mathrm{X} ; \hat{\xi}]
$$

En general, este valor esperado condicional es imposible de resolver analíticamente, por lo que se usa el método de simulaciones de Montecarlo para obtener un estimador $\hat{H}_{c}^{E}$. El problema se repite para el cálculo de los errores estándar, ya que dicho cálculo no es trivial y, de hecho, no es posible calcularlos analíticamente. Por esta razón, se debe recurrir al método de bootstrapping y simulaciones de Montecarlo para su estimación.

${ }^{11}$ Aplicando el teorema del límite central se asume que la distribución del vector $\beta$ converge a una distribución normal multivariada. 
La diferencia entre $\tilde{H}_{c}^{E}$, el estimador del valor esperado de $H$, y el nivel actual de $H$ para la zona geográfica se puede descomponer en tres partes de la siguiente forma:

$$
H_{c}-\tilde{H}_{c}^{E}=\left(H_{c}-H_{c}^{E}\right)+\left(H_{c}^{E}-\hat{H}_{c}^{E}\right)+\left(\hat{H}_{c}^{E}-\tilde{H}_{c}^{E}\right)
$$

Es así como el error de predicción tiene tres componentes, el error idiosincrático, el error del modelo y el error de cálculo. El primero se debe a la presencia de un error estocástico en el modelo de la primera etapa, lo cual implica que los ingresos actuales de los hogares se desvían de sus valores esperados; el segundo se debe a la varianza en los estimadores de la primera etapa de los parámetros del modelo; y el tercero se debe a un método inexacto para calcular $\hat{H}_{c}^{E}$.

Debido al error idiosincrático, la varianza del estimador disminuye en forma inversamente proporcional al tamaño de la población para cada zona geográfica. Mientras más pequeño sea el nivel de desagregación geográfica, mayor será el primer componente de error generando restricciones al nivel de desagregación posible ${ }^{12}$.

La varianza del estimador debida al error del modelo puede calcularse usando el método delta: $V_{\text {modelo }}=\nabla^{T} V(\xi) \nabla$ donde $\nabla=\left[\partial H^{E} / \partial \xi\right]$, $V(\xi)$ es la matriz de varianzas y covarianzas de los estimadores de la primera etapa y $\xi$ es un estimador consistente de $\xi$ también obtenido en la primera etapa. Este componente de la varianza del estimador de pobreza está determinado por las propiedades de los estimadores de la primera etapa y por esta razón no cambia sistemáticamente con el tamaño de la zona geográfica. Su magnitud depende exclusivamente de la precisión de los parámetros estimados en la primera etapa.

El error de cálculo de la varianza del estimador depende del método computacional utilizado. Dado que el método es una simulación, es posible reducir este componente al mínimo por medio de un número grande de simulaciones. La mayoría de los trabajos que han implementado esta metodología usan cien simulaciones; sin embargo,

$$
{ }^{12} \text { El componente idiosincrático del error es igual a } \Sigma \nu_{N} \text {, donde } \mathrm{S}=\frac{E\left[n_{c}^{2} \operatorname{Var}\left(G / X_{c}, \beta\right)\right]}{(E[n])^{2}} .
$$

Por esta razón, el componente del error aumenta al desagregar el indicador de desigualdad en poblaciones más pequeñas. 
en este trabajo se han usado 250 para reducir aún más este componente del error ${ }^{13}$.

Se utilizaron simulaciones de Montecarlo para calcular el valor esperado de $H$ condicional en la regresión de la primera etapa, la varianza generada por el componente idiosincrático del ingreso per cápita de los hogares y el vector de gradientes. Cada simulación obtiene 250 vectores de términos de error de la distribución estimada en la primera etapa, con cada uno de estos vectores se calcula un valor para $H$ y luego el valor esperado simulado para $H$ es la media para las 250 réplicas:

$$
\tilde{H}^{E}=\frac{1}{250} \sum_{d=1}^{250} \tilde{H}_{d}^{E}
$$

De la misma manera, la varianza de $H$ es estimada usando:

$$
\hat{V}_{\text {mod elo }}=\frac{1}{250} \sum_{d=1}^{250}\left(H_{d}-\tilde{H}^{E}\right)^{2} .
$$

Es importante destacar que un supuesto fundamental de esta metodología es que los modelos econométricos estimados usando los datos de encuestas son aplicables a las observaciones del censo. Este supuesto es bastante razonable si el año del censo y la encuesta coinciden o son bastante cercanos, como ocurre en el caso de este trabajo, en que se utiliza la encuesta Casen del año 2003 y el censo del año 2002.

13 Aumentar el número de simulaciones sobre 250 no tuvo ningún impacto relevante en la magnitud de los errores estándar. 


\section{ANEXO}

GRÁFICO 1A: $\quad$ DISTRIBUCIÓN DEL PORCENTAJE DE VIVIENDAS CON PARED DE LADRILLO O CEMENTO EN LA RM (1992 Y 2002)
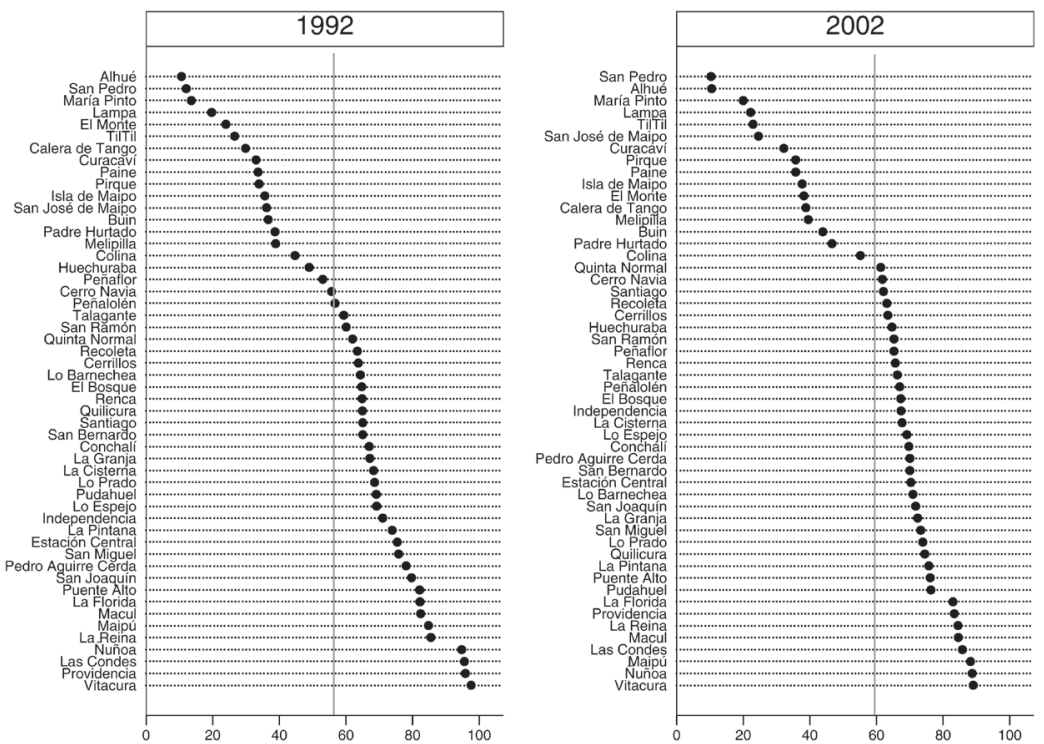

Fuente: Censo Población y Vivienda, 1992 y 2002.

GRÁFICO 2A: DISTRIBUCIÓN DEL PORCENTAJE DE VIVIENDAS CON PARED DE ADOBE EN LA RM (1992 Y 2002)
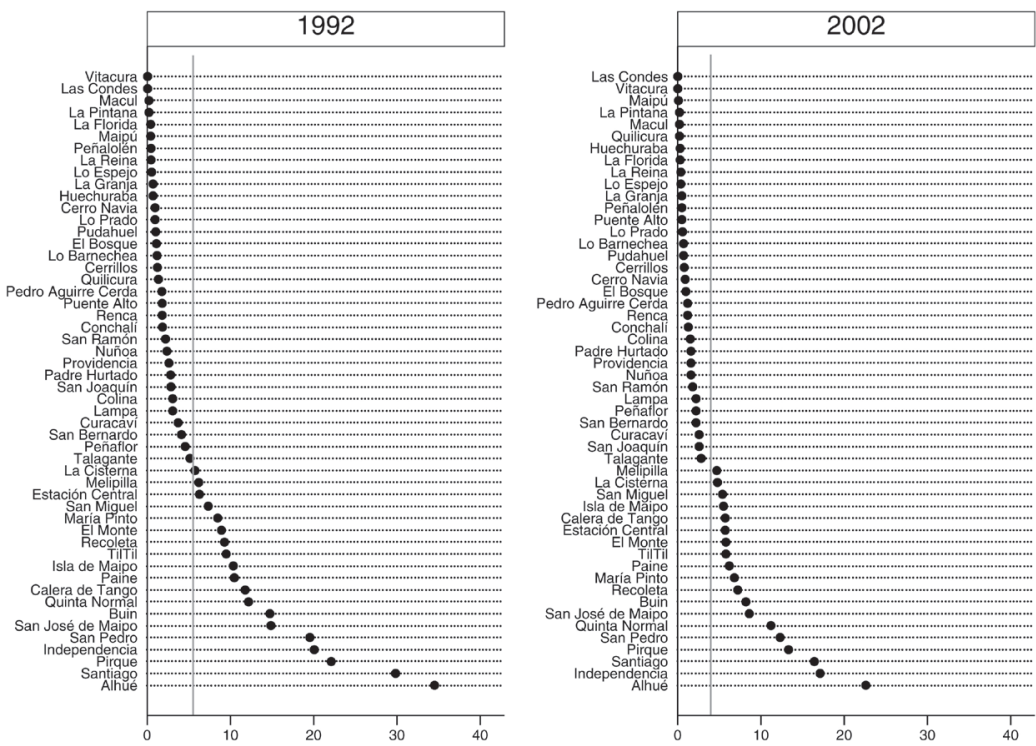

Fuente: Censo Población y Vivienda, 1992 y 2002. 
GRÁFICO 3A: DISTRIBUCIÓN DEL PORCENTAJE DE VIVIENDAS CON PISO DE TIERRA EN LA RM (1992 Y 2002)
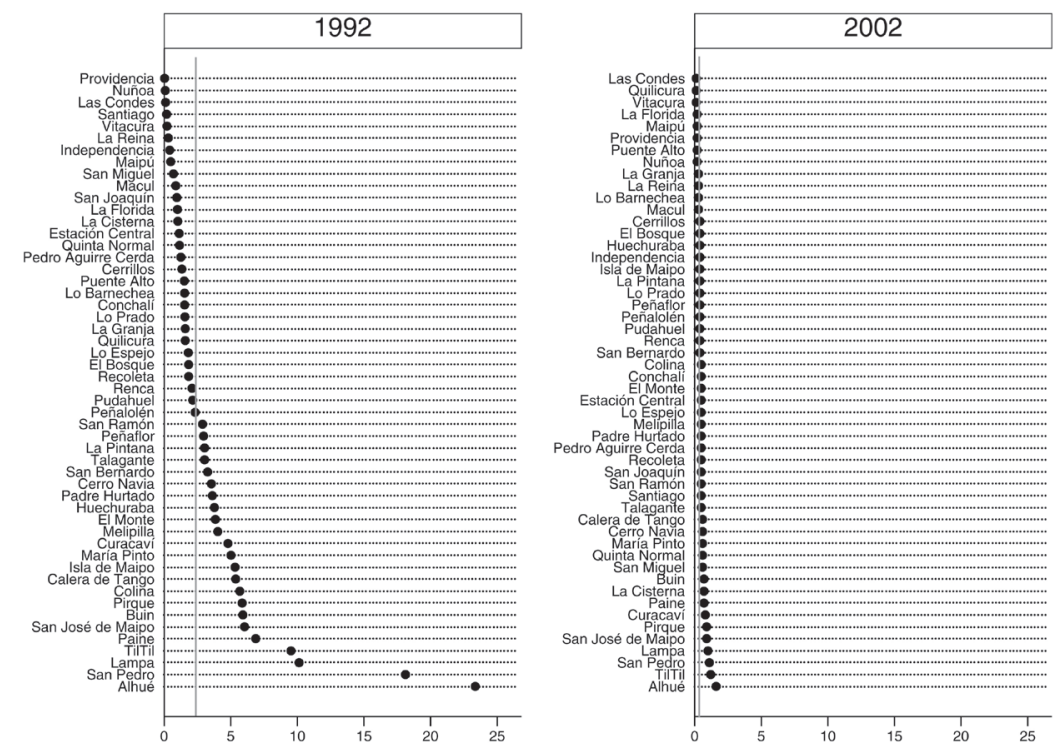

Fuente: Censo Población y Vivienda, 1992 y 2002.

GRÁFICO 4A: DISTRIBUCIÓN DEL PORCENTAJE DE VIVIENDAS CON PISO DE BALDOSÍN EN LA RM (1992 Y 2002)
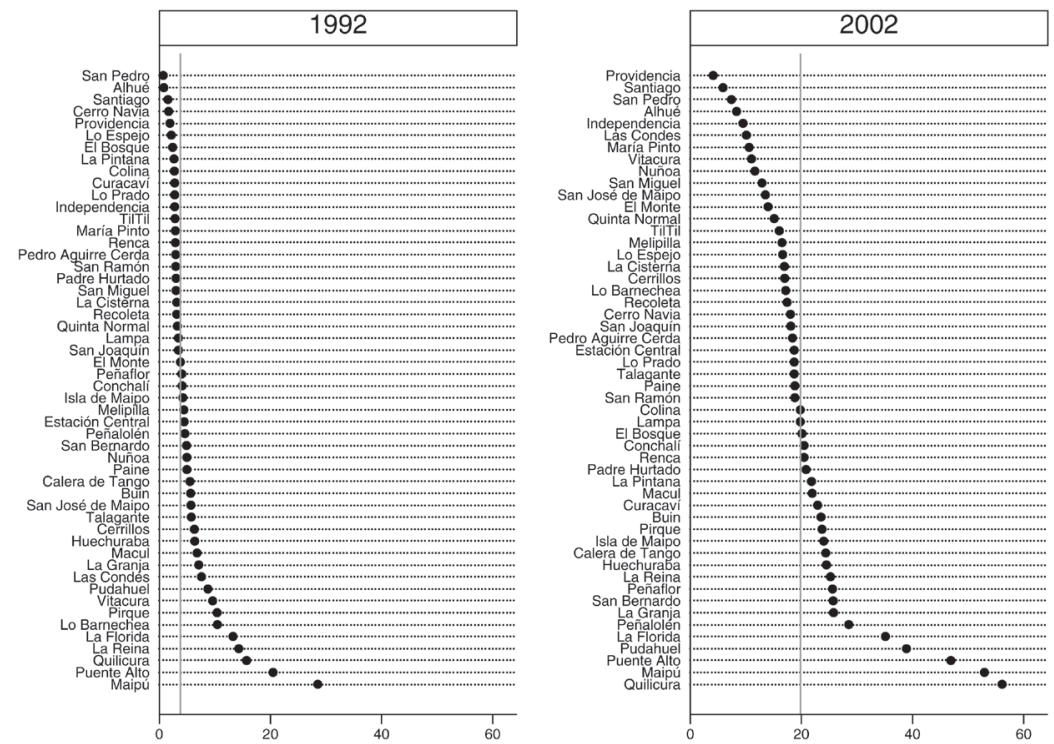

Fuente: Censo Población y Vivienda, 1992 y 2002. 
GRÁFICO 5A: $\quad$ DISTRIBUCIÓN DEL PORCENTAJE DE VIVIENDAS CON TECHO DE TEJUELAS EN LA RM (1992 Y 2002)
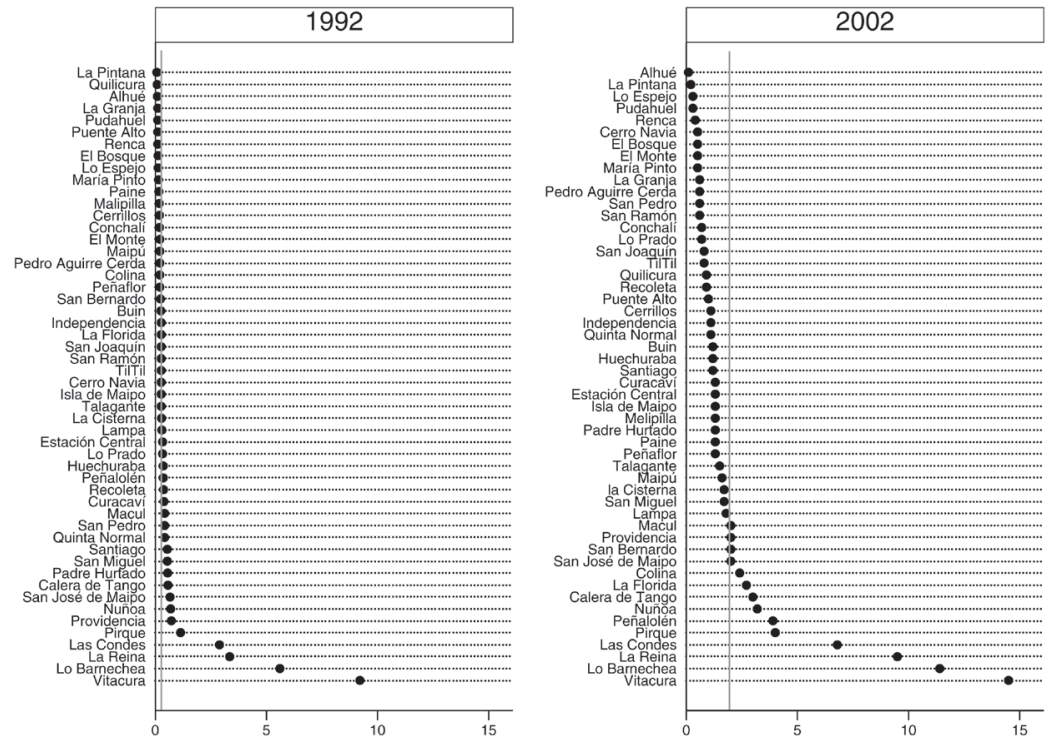

Fuente: Censo Población y Vivienda, 1992 y 2002.

GRÁFICO 6A: DISTRIBUCIÓN DEL PORCENTAJE DE VIVIENDAS CON TECHO DE OTROS MATERIALES (COMO FONOLITA, FIBRA VIDRIO, PAJA EMBARRADA) EN LA RM (1992 Y 2002)
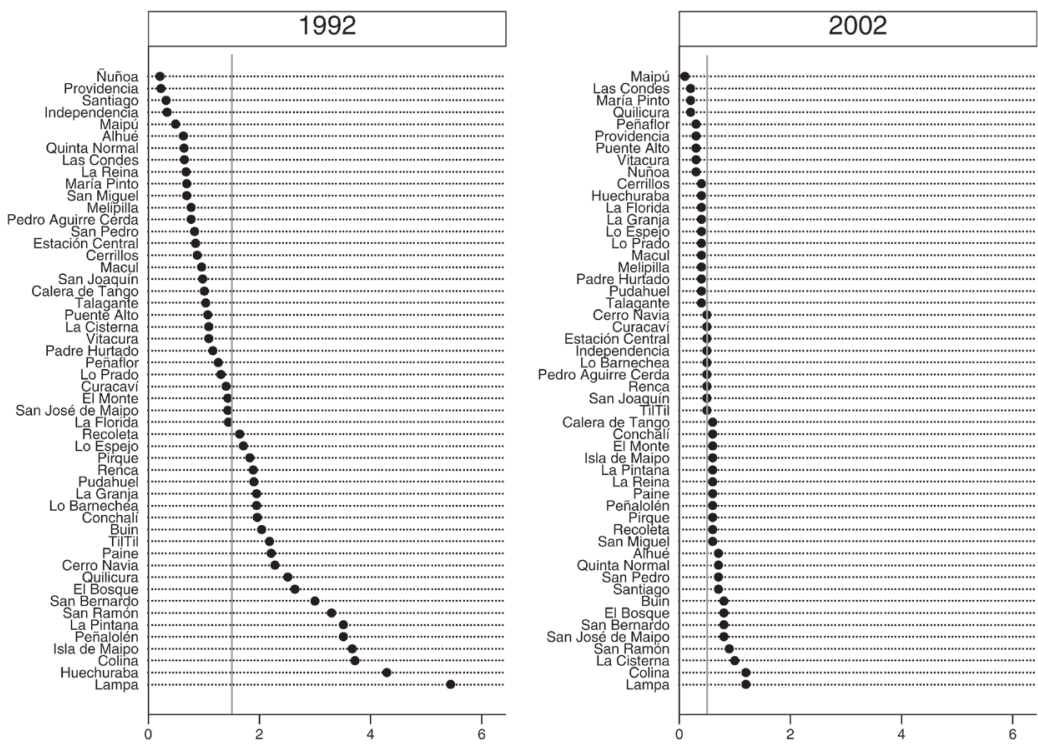

Fuente: Censo Población y Vivienda, 1992 y 2002. 
GRÁFICO 7A: $\quad$ DISTRIBUCIÓN DEL PORCENTAJE DE VIVIENDAS DE TIPO CASA EN LA RM (1992 Y 2002)
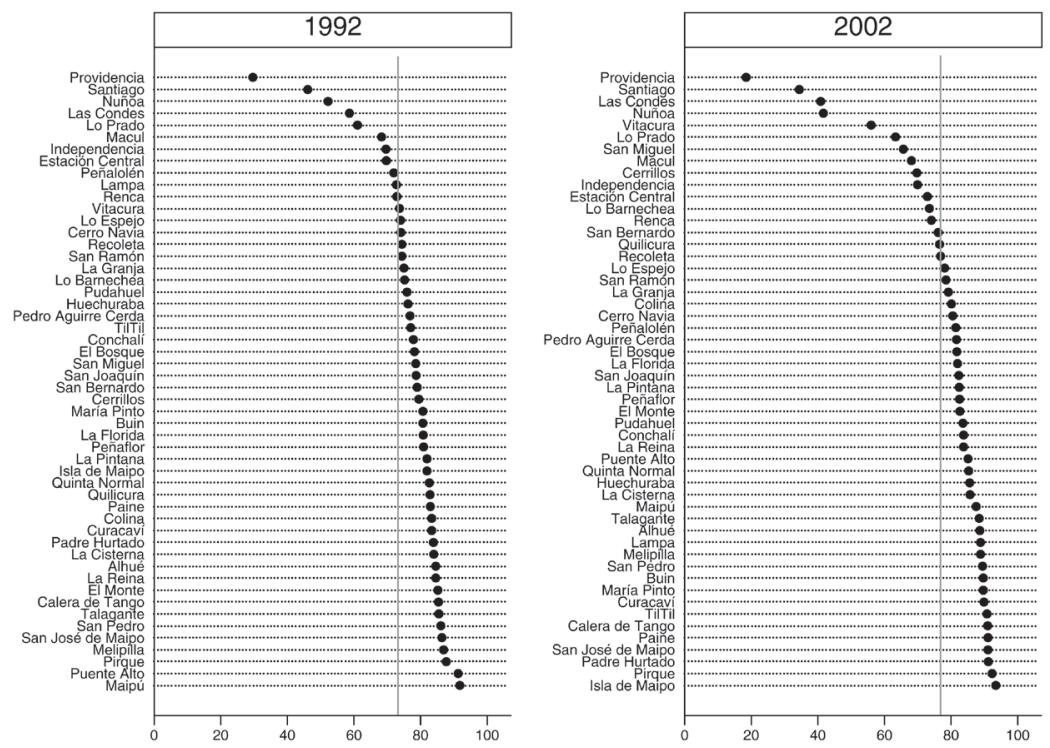

Fuente: Censo Población y Vivienda, 1992 y 2002.

GRÁFICO 8A: DISTRIBUCIÓN DEL PORCENTAJE DE VIVIENDAS DE TIPO DEPARTAMENTO EN LA RM (1992 Y 2002)
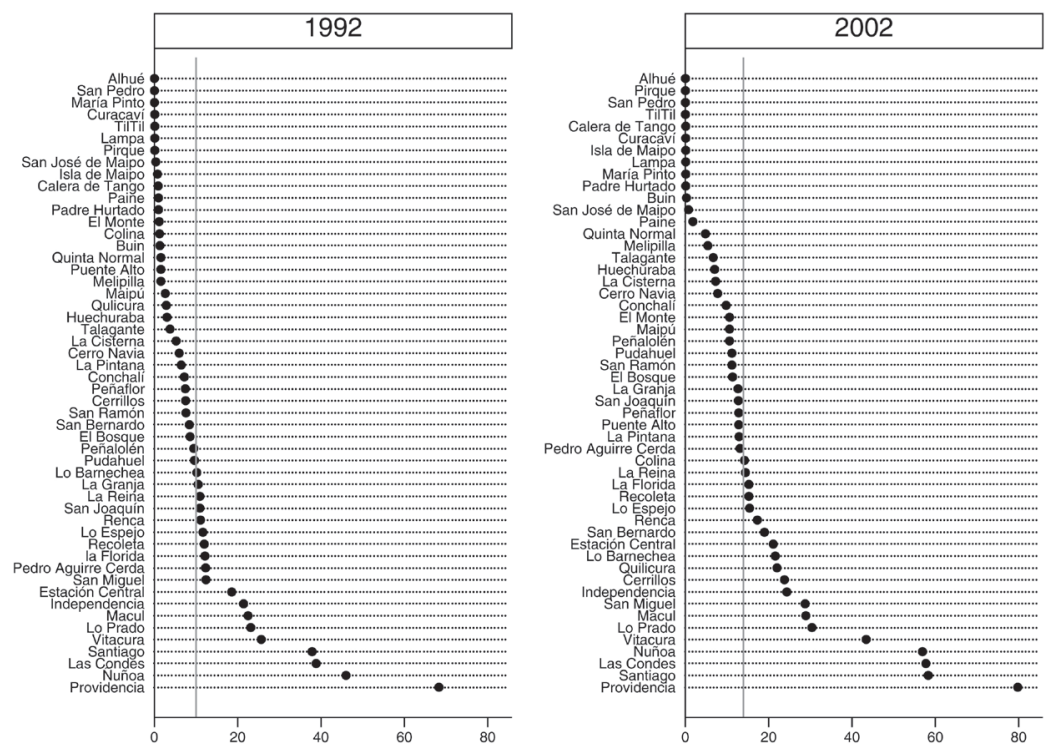

Fuente: Censo Población y Vivienda, 1992 y 2002. 
GRÁFICO 9A:

DISTRIBUCIÓN DEL PORCENTAJE DE VIVIENDAS DE TIPO MEDIAGUA EN LA RM (1992 Y 2002)
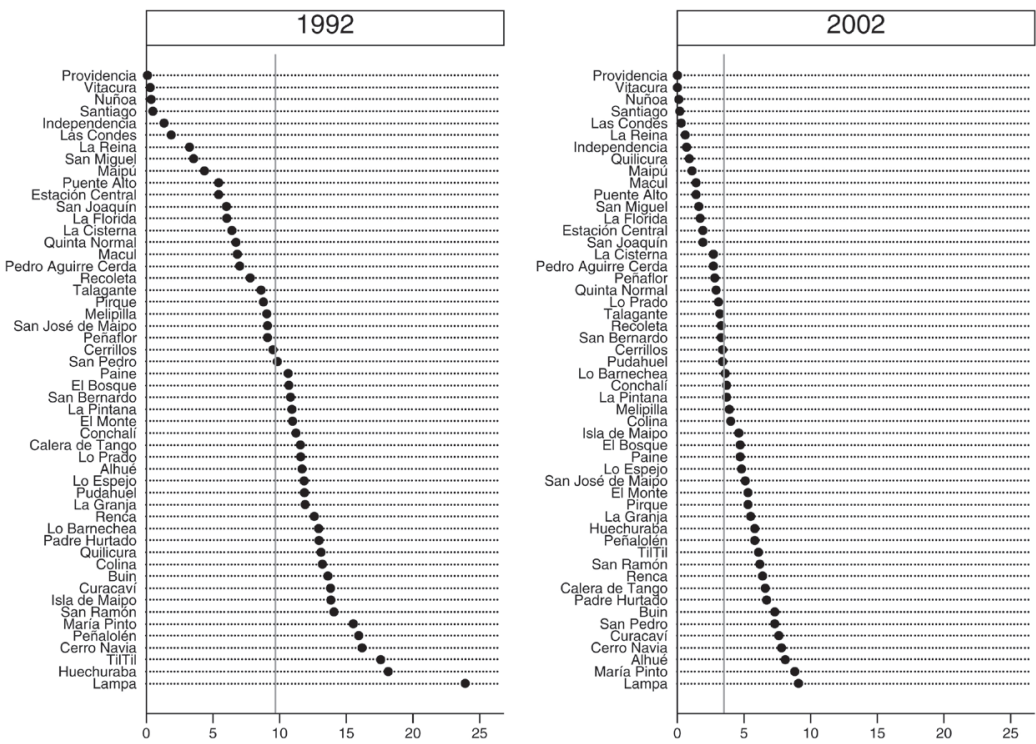

Fuente: Censo Población y Vivienda, 1992 y 2002.

GRÁFICO 10A: DISTRIBUCIÓN DEL PORCENTAJE DE HOGARES QUE TIENEN REFRIGERADOR EN LA RM (1992 Y 2002)
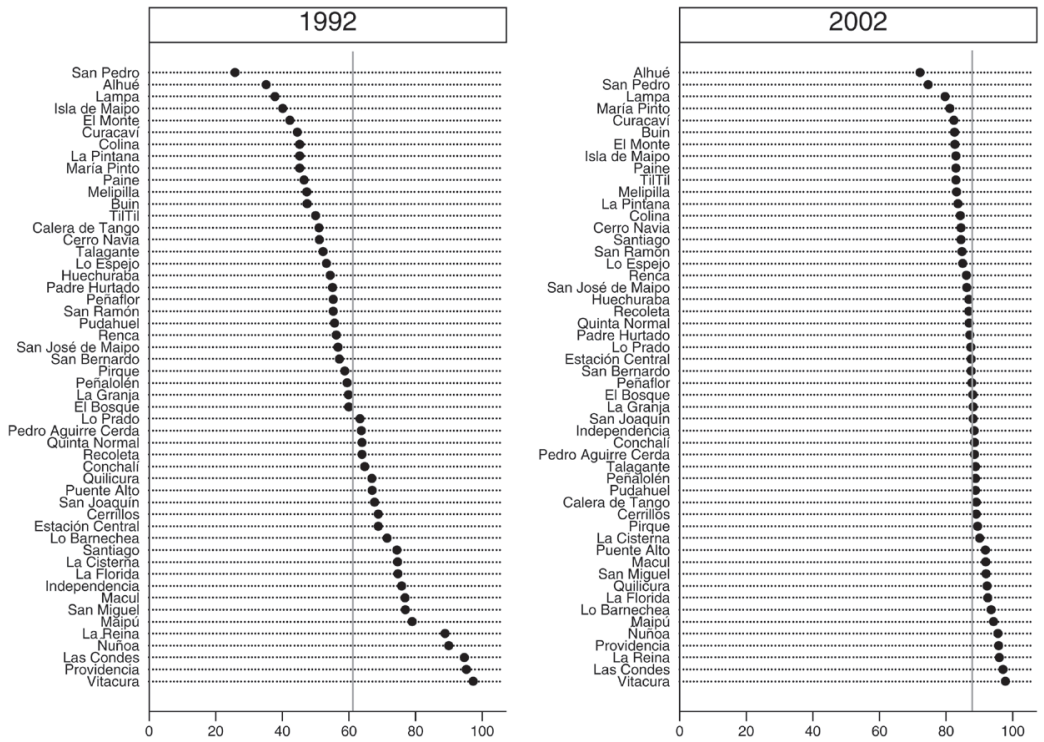

Fuente: Censo Población y Vivienda, 1992 y 2002. 
GRÁFICO 11A: DISTRIBUCIÓN DEL PORCENTAJE DE HOGARES QUE TIENEN TELEVISOR EN COLORES EN LA RM (1992 Y 2002)
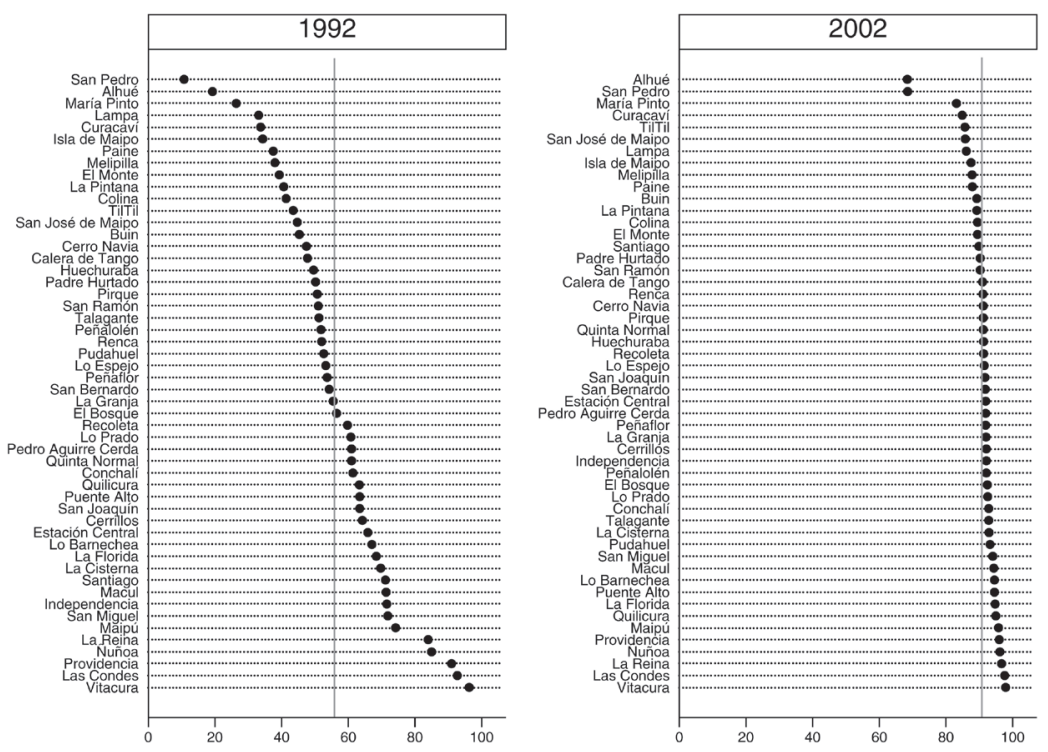

Fuente: Censo Población y Vivienda, 1992 y 2002.

GRÁFICO 12A: DISTRIBUCIÓN DEL PORCENTAJE DE HOGARES QUE TIENEN VIDEOGRABADOR EN LA RM (1992 Y 2002)
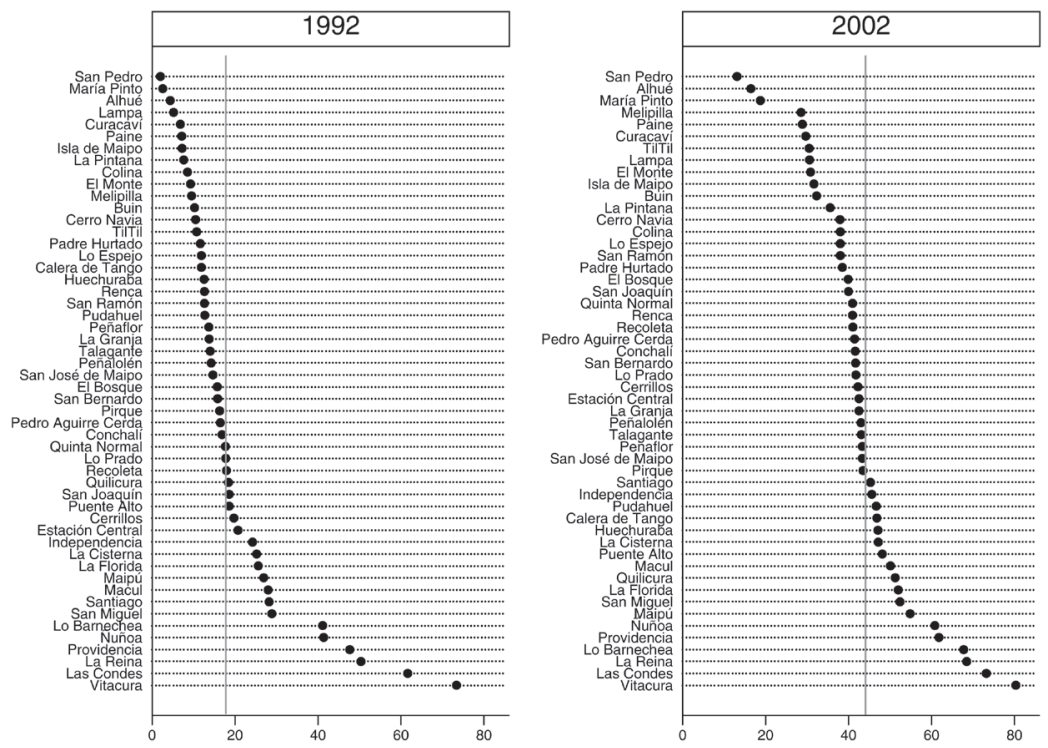

Fuente: Censo Población y Vivienda, 1992 y 2002. 
GRÁFICO 13A: DISTRIBUCIÓN DEL PORCENTAJE DE HOGARES QUE TIENEN LAVADORA EN LA RM (1992 Y 2002)
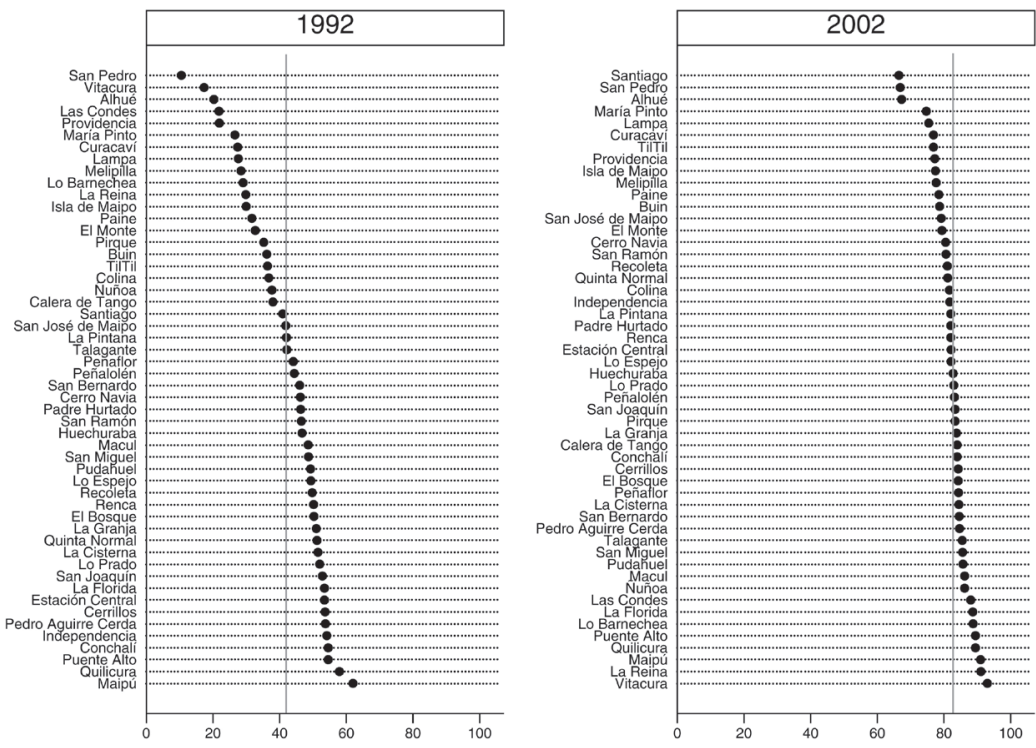

Fuente: Censo Población y Vivienda, 1992 y 2002.

GRÁFICO 14A: DISTRIBUCIÓN DEL PORCENTAJE DE HOGARES QUE TIENEN HORNO MICROONDAS EN LA RM (1992 Y 2002)
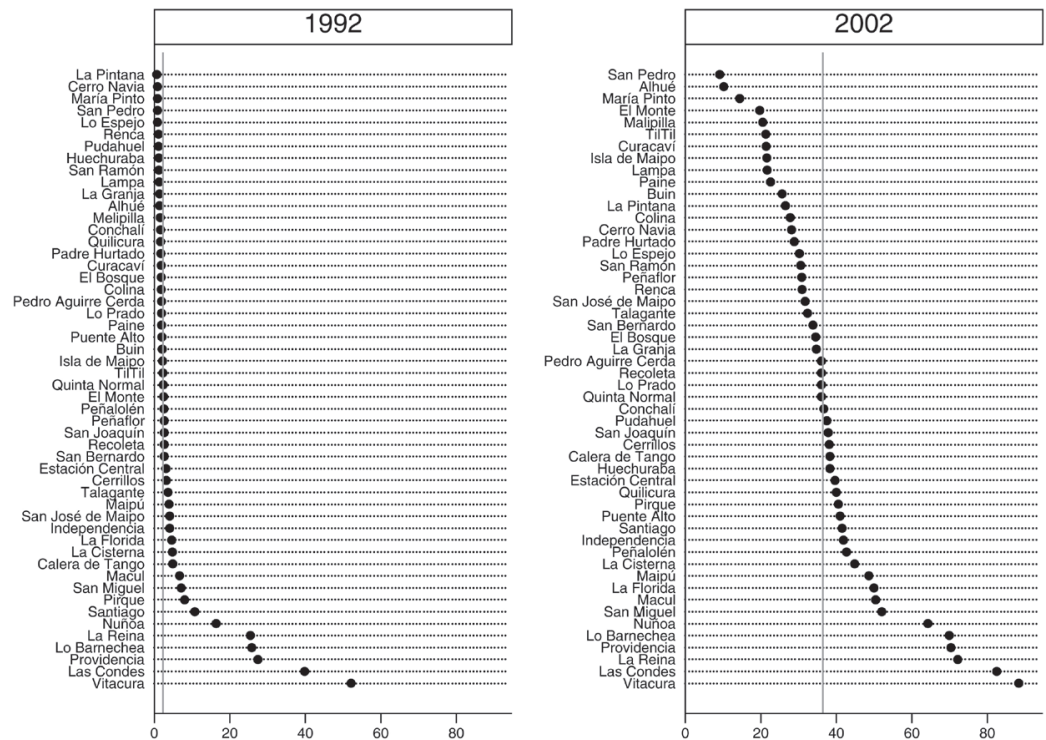

Fuente: Censo Población y Vivienda, 1992 y 2002. 
GRÁFICO 15A: DISTRIBUCIÓN DEL PORCENTAJE DE HOGARES QUE TIENEN CELULAR EN LA RM (1992 Y 2002)
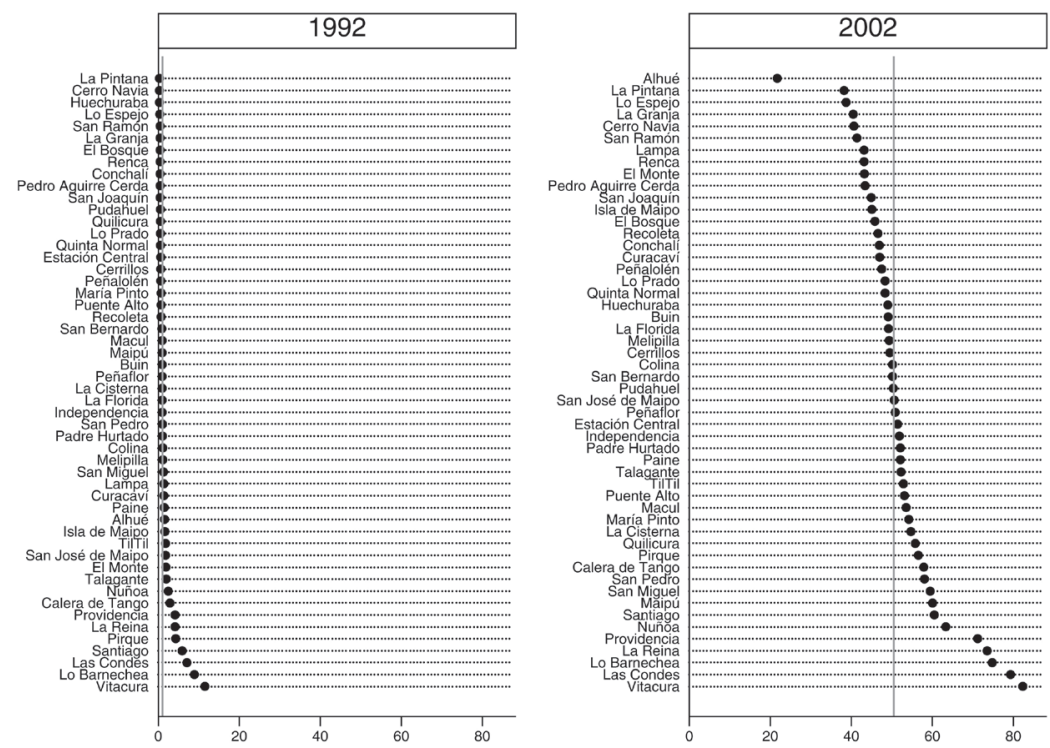

Fuente: Censo Población y Vivienda, 1992 y 2002.

GRÁFICO 16A: DISTRIBUCIÓN DEL PORCENTAJE DE HOGARES QUE TIENEN TELÉFONO FIJO EN LA RM (1992 Y 2002)
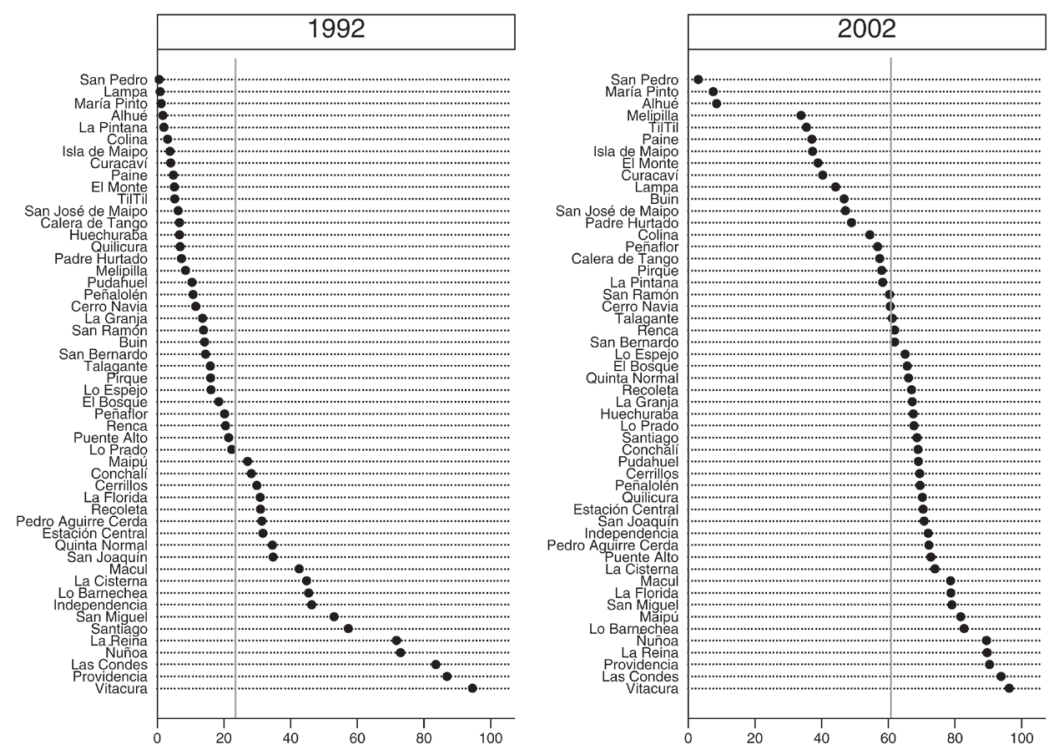

Fuente: Censo Población y Vivienda, 1992 y 2002. 
GRÁFICO 17A: DISTRIBUCIÓN DEL PORCENTAJE DE JEFES DE HOGAR CON EDUCACIÓN BÁSICA COMPLETA EN LA RM (1992 Y 2002)
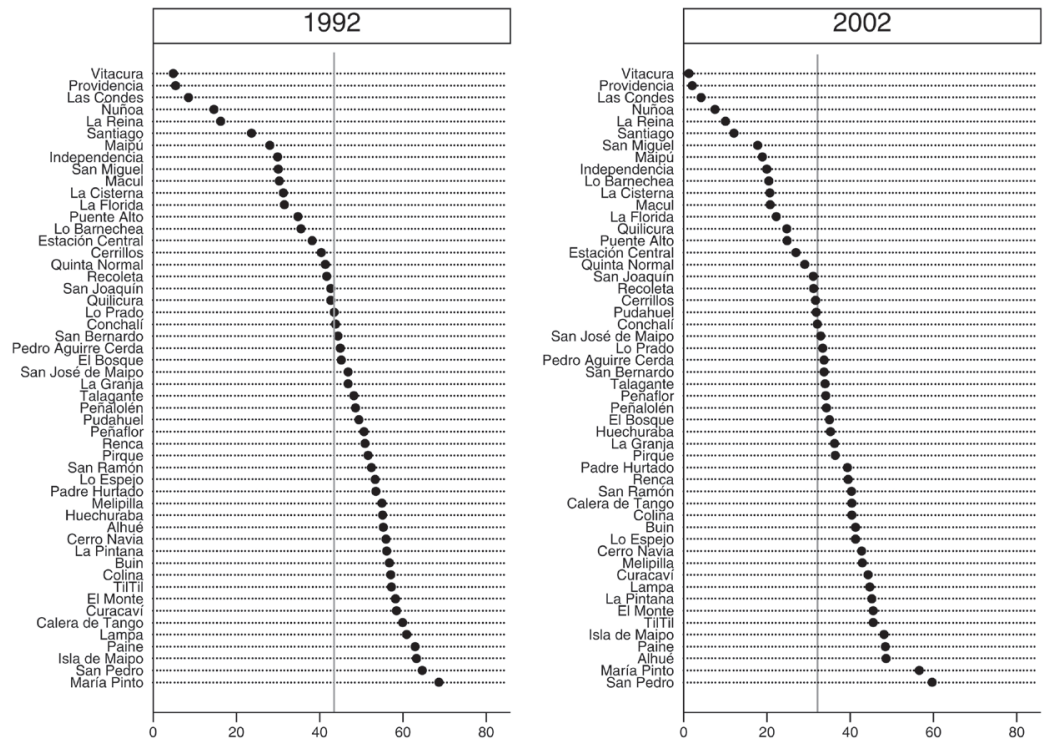

Fuente: Censo Población y Vivienda, 1992 y 2002.

GRÁFICO 18A: DISTRIBUCIÓN DEL PORCENTAJE DE JEFES DE HOGAR CON EDUCACIÓN MEDIA COMPLETA EN LA RM (1992 Y 2002)
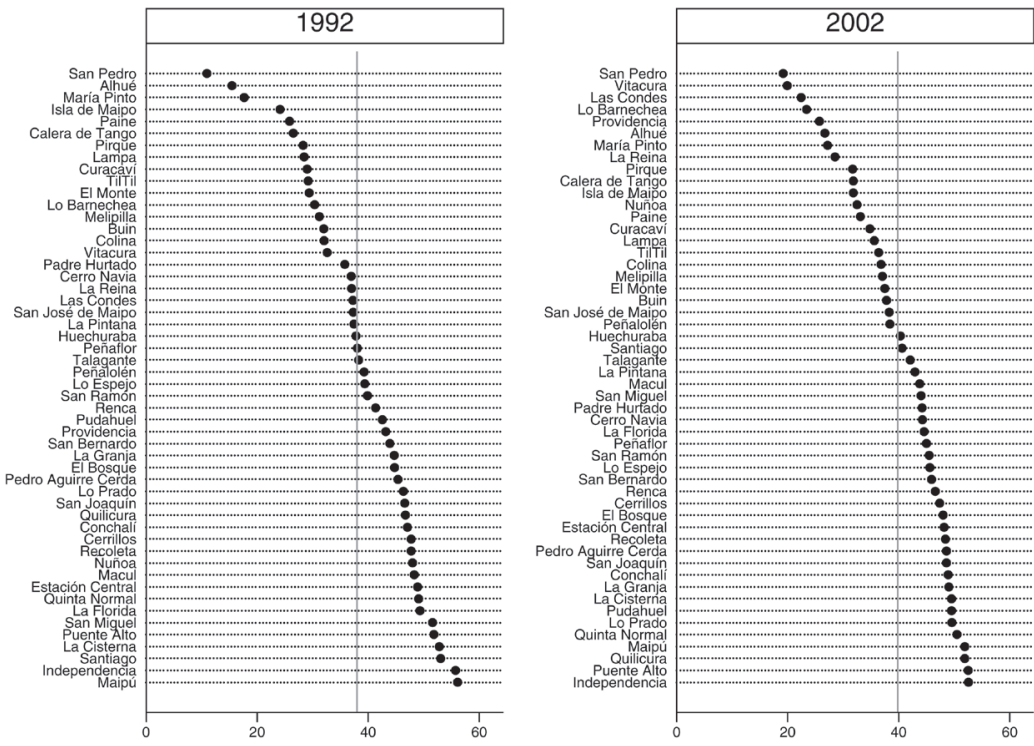

Fuente: Censo Población y Vivienda, 1992 y 2002. 
GRÁFICO 19A: DISTRIBUCIÓN DEL PORCENTAJE DE JEFES DE HOGAR CON FORMACIÓN TÉCNICA COMPLETA EN LA RM (1992 Y 2002)
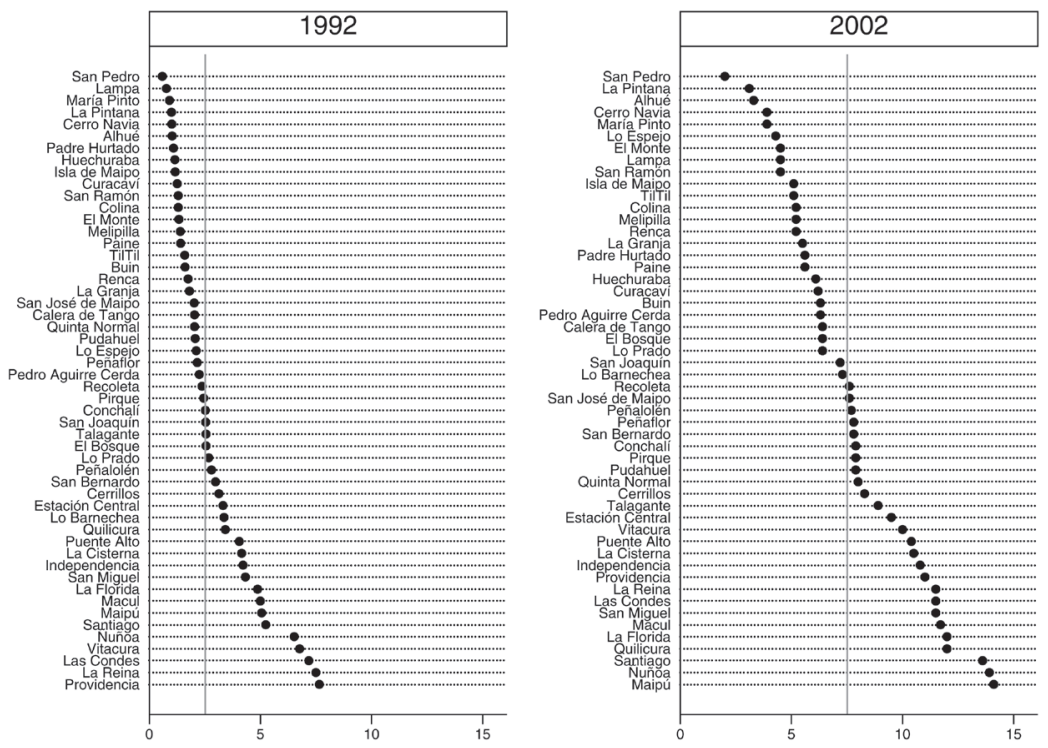

Fuente: Censo Población y Vivienda, 1992 y 2002.

GRÁFICO 20A: DISTRIBUCIÓN DEL PORCENTAJE DE JEFES DE HOGAR CON EDUCACIÓN UNIVERSITARIA COMPLETA EN LA RM (1992 Y 2002)
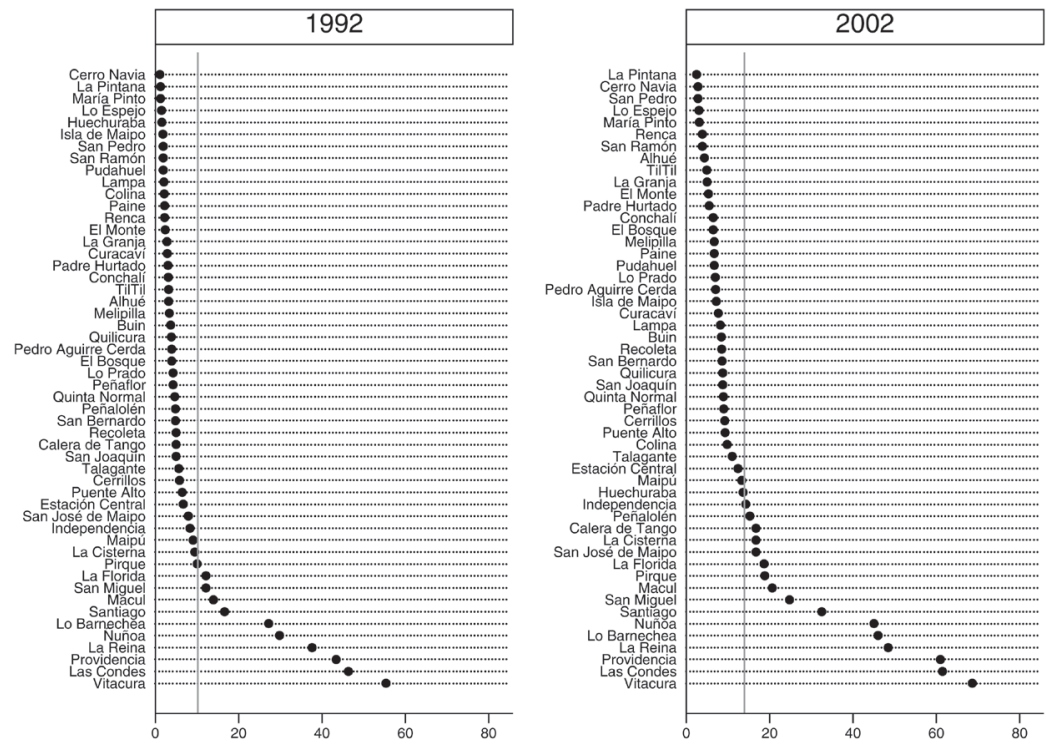

Fuente: Censo Población y Vivienda, 1992 y 2002. 
GRÁFICO 21A: DISTRIBUCIÓN DEL PORCENTAJE DE POBLACIÓN FEMENINA CON EMPLEO EN LA RM (1992 Y 2002)
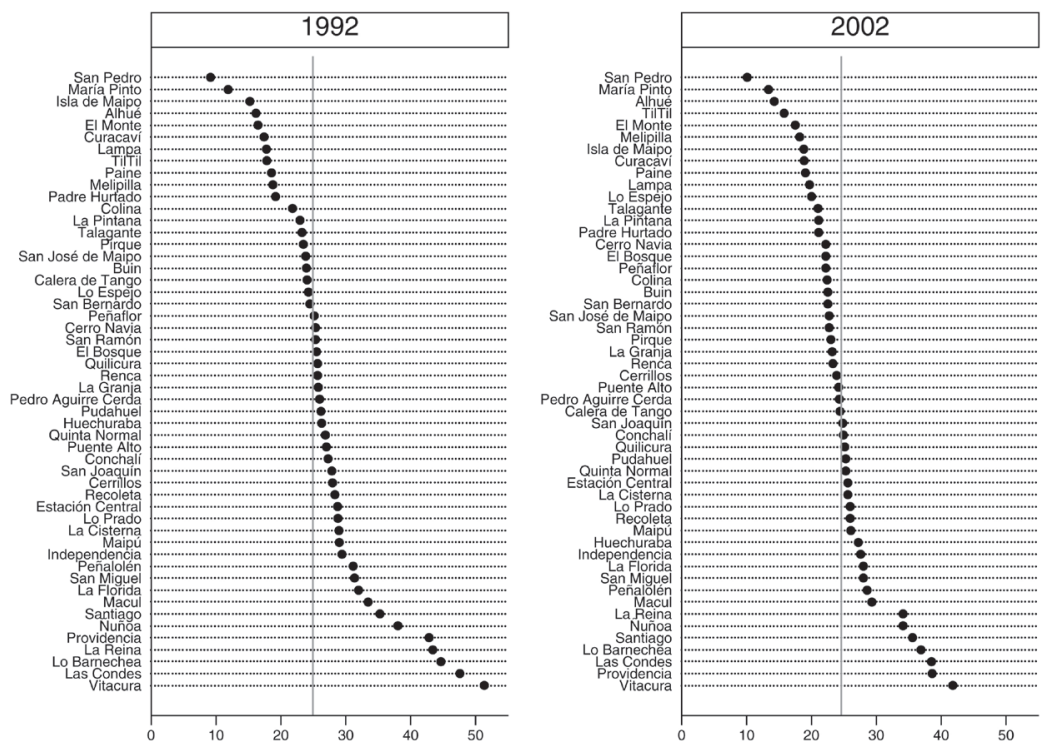

Fuente: Censo Población y Vivienda, 1992 y 2002.

\section{REFERENCIAS}

Agostini, C. A. y P. H. Brown: "Desigualdad Geográfica en Chile". Revista de Análisis Económico, 22(1): 3-33, 2007.

"Distribución Espacial de la Pobreza en Chile". Estudios de Economía, 35(1): 79-110, 2008.

Agostini, C. A., P. H. Brown y D. P. Góngora: "Public Finance, Governance, and Cash Transfers in Alleviating Poverty and Inequality in Chile". "Public Budgeting and Finance" (2010, en prensa)

Atkinson, A. B.: "Poverty". En Steven N. Durlauf y Lawrence E. Blume (eds.), The New Palgrave Dictionary of Economics. Palgrave Macmillan, segunda edición, 2008.

Bayer, P., R. McMillan y K. Reuben: "What Drives Racial Segregation". Journal of Urban Economics, 56, 514-535, 2004.

Bell, W.: "A Probability Model for the Measurement of Ecological Segregation". Social Forces, 43: 357-364, 1954.

Burton, E.: "Housing for an Urban Renaissance: Implications for Social Equity". Housing Studies 18(4), 537-562, 2003.

Calabrese, S., D. Epple, et al.: "Local Public Good Provision: Voting, Peer Effects, and Mobility”. Journal of Public Economics 90(6-7): 959-981, 2006. 
Charles, C., G. Dinwiddie y D. Massey: "The Continuing Consequences of Segregation: Family Stress and College Academic Performance”. Social Sciences Quarterly, 85(5), 1353-1373, 2004.

Clapp, J. y S. Ross: "Schools and Housing Markets: an Examination of School Segregation and Performance in Connecticut”. Economic Journal 114(499), 425-440, 2004.

Cutler, D. y E. Glaeser: “Are Ghettos Good or Bad?” Quarterly Journal of Economics 112(3), 826-872, 1997.

Cutler, D., E. Glaeser y J. Vigdor: "The Rise of the American Ghetto". Journal of Political Economy, 107(3), 455-505, 1999.

Dawkings, J., Q. Shen y T. Sanchez: "Race, Space, and Unemployment Duration”. Journal of Urban Economics, 58, 91-113, 2005.

Duncan, O. D. y B. Duncan: “A Methodological Analysis of Segregation Indexes". American Sociological Review 20, 1955.

Elbers, Chris, Jean O. Lanjouw y Peter Lanjouw: "Micro-Level Estimation of Poverty and Inequality”. Econometrica 71(1), 2003.

Epple, D. y G. J. Platt: "Equilibrium and Local Redistribution in an Urban Economy When Households Differ in both Preferences and Income”. Journal of Urban Economics, 43, 23-51, 1998.

Epple, D. y H. Sieg: “Estimating Equilibrium Models of Local Jurisdictions”. The Journal of Political Economy, 107(4): 645-681, 1999.

Erbe, B.: "Race and Socioeconomic Segregation”. American Sociological Review, 40(6): 801-812, 1975.

Fossett, M.: "Measuring Uneven Distribution: Conceptual and Practical Considerations for Segregation Studies". Paper presented at the annual meeting of the American Sociological Association, Marriott Hotel, Loews Philadelphia Hotel, Philadelphia, PA, 2005.

Galster, G.: "Housing Discrimination and Urban Poverty of African-Americans". Journal of Housing Research, 2(2), 1991.

"Research on Discrimination in Housing and Mortgage Markets: Assessment and Future Directions”. Housing Policy Debate 3(2): 639-83, 1992.

Galster, G. C. y S. P. Killen: "The Geography of Metropolitan Opportunity: A Reconnaissance and Conceptual Framework”. Housing Policy Debate, 6(1), 744, 1995.

Glaeser, E. L.: "Inequality”. Working Paper No 11511, National Bureau of Economic Research, 2005.

Hanushek, E. y K. Yilmaz: “The Complementarity of Tiebout and Alonso”. Journal of Housing Economics, 16(2): 243-261, 2007.

Harsman, B. y J. Quigley: “The Spatial Segregation of Ethnic and Demographic Groups: Comparative Evidence from Stockholm and San Francisco". Journal of Urban Economics, 37, 1-16, 1995.

Hoxby, C. M.: "The Productivity of Schools and Other Local Public Goods Producers”. Journal of Public Economics, 74(1): 1-30, 1999. -: "Does Competition among Public Schools Benefit Students and Taxpayers?" American Economic Review, 90(5): 1209-1238, 2000.

Hutchens, R. M.: "Segregation Curves, Lorenz Curves, and Inequality in the Distribution of People across Occupations”. Mathematical Social Sciences, 21(1): 31-51, 1991. 
Kremer, M. y E. Maskin: “Wage Inequality and Segregation by Skill”. NBER Working Paper Nº W5718, 1996.

Larrañaga, O. y C. Sanhueza: "Residential Segregation Effects on Poor’s Opportunities in Chile”. Documento de Trabajo $\mathrm{N}^{\circ}$ 259, Departamento de Economía, Universidad de Chile, 2007.

Logan, J. y S. Messner: "Racial Residential Segregation and Suburban Violent Crime”. Social Sciences Quarterly, 68(3), 510-527, 1987.

Massey, D. S. y N. A. Denton: “The Dimensions of Residential Segregation”. Social Forces, 67(2), 281-315, 1988.

Miller, V. y J. Quigley: “Segregation by Racial and Demographic Group: Evidence from the San Francisco Bay Area”. Urban Studies, 27, 3-21, 1990.

Nechyba, T.: "School Finance Induced Migration and Stratification Patterns: The Impact of Private School Vouchers”. Journal of Public Economic Theory, 1(1), 5-50, 1999.

Rodríguez, J.: “Segregación Residencial Económica: ¿Qué es? ¿Cómo se Mide? ¿Qué está Pasando? ¿Importa?”. Población y Desarrollo, 16, 2001.

Ross, N.A., K. Nobrega y J. Dunn: "Income Segregation, Income Inequality and Mortality in North American Metropolitan Areas”. GeoJournal 53(2), 2002.

Sabatini, F.: “Tendencias de la Segregación Residencial Urbana en Latinoamérica: Reflexiones a Partir del Caso de Santiago de Chile”. Presentado en Seminario: Latin American: Democracy, Markets and Equity at the Threshold of New Millenium. Universidad de Upsala, Suecia, 1999.

"Reforma de los Mercados de Suelo en Santiago, Chile: Efectos sobre los Precios de la Tierra y la Segregación Residencial”. EURE, 26(77): 49-80, 2000.

- "Medición de la Segregación Residencial: Reflexiones Metodológicas desde la Ciudad Latinoamericana”. En G. Cáceres y F. Sabatini, Barrios Cerrados en Santiago de Chile: Entre la Exclusión y la Integración Residencial. Santiago de Chile: Instituto de Geografía, PUC Chile, 2004.

"The Social Spatial Segregation in the Cities of Latin America”. Washington DC: Inter-American Development Bank, Sustainable Development Department, 2006.

Sabatini, F., G. Cáceres y J. Cerda: “Segregación Residencial en las Principales Ciudades Chilenas: Tendencias en las Últimas Tres Décadas y Posibles Cursos de Acción”. EURE, 27(82), 21-42, 2001.

Schafer, R.: "Racial's Discrimination in the Boston Housing Market". Journal of Urban Economics, 6, 1176-1196, 1979.

Sethi, R. y R. Somanathan: "Inequality and Segregation”. Journal of Political Economy, 112(6), 2004.

Tiebout, C. M.: “A Pure Theory of Local Expenditures”. The Journal of Political Economy, 64(5): 416-424, 1956.

Van Valey, T. L. y W. C. Roof: “Measuring Segregation in American Cities”. Urban Affairs Quarterly, 11, 453-568, 1976.

Vandell, Kerry D.: "Market Factors Affecting Spatial Heterogeneity among Urban Neighborhoods”. Housing Policy Debate, 6(1), 103-139, 1995.

Vargas, M.: "Causes of Residential Segregation: The Case of Santiago, Chile”. Mimeo, Centre for Spatial and Real Estate Economics, Department of Economics, The University of Reading, United Kingdom, 2006.

Vargas, M. y V. Royuela: “Segregación Residencial: Una Revisión de la Literatura”. Mimeo, Facultad de Economía y Empresa, Universidad Diego Portales, 2007. 
Vigdor, Jacob L.: “Residential Segregation”. En Steven N. Durlauf y Lawrence E. Blume (eds.), The New Palgrave Dictionary of Economics. Palgrave Macmillan, segunda edición, 2008.

Yinger, J.: "The Black-White Price Differentials in Housing: Some further Evidence", Land Economics 54, 1978.

White, M. J.: "The Measurement of Spatial Segregation". American Journal of Sociology, 88(5), 1008-1018, 1983. 